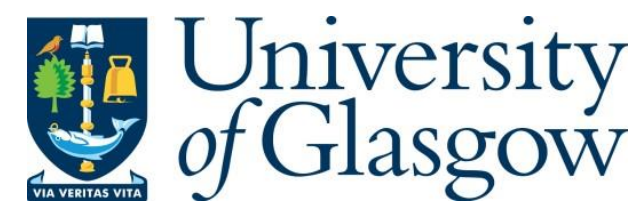

Gadre, V. (2017) Partial sums of excursions along random geodesics and volume asymptotics for thin parts of moduli spaces of quadratic differentials. Journal of the European Mathematical Society, 19(10), pp. 3053-3089. (doi:10.4171/JEMS/735)

This is the author's final accepted version.

There may be differences between this version and the published version. You are advised to consult the publisher's version if you wish to cite from it.

http://eprints.gla.ac.uk/126743/

Deposited on: 21 September 2016

Enlighten - Research publications by members of the University of Glasgow http://eprints.gla.ac.uk 


\title{
PARTIAL SUMS OF EXCURSIONS ALONG RANDOM GEODESICS AND VOLUME ASYMPTOTICS FOR THIN PARTS OF MODULI SPACES OF QUADRATIC DIFFERENTIALS.
}

\author{
VAIBHAV GADRE
}

\begin{abstract}
For a non-uniform lattice in $S L(2, \mathbb{R})$, we consider excursions of a random geodesic in cusp neighborhoods of the quotient finite area hyperbolic surface or orbifold. We prove a strong law for a certain partial sum involving these excursions. This generalizes a theorem of Diamond and Vaaler for continued fractions [9]. In the Teichmüller setting, we consider invariant measures for the $S L(2, \mathbb{R})$ action on the moduli spaces of quadratic differentials. By the work of Eskin and Mirzakhani [12], these measures are supported on affine invariant submanifolds of a stratum of quadratic differentials. For a Teichmüller geodesic random with respect to a $S L(2, R)$-invariant measure, we study its excursions in thin parts of the associated submanifold. Under a regularity hypothesis for the invariant measure, we prove similar strong laws for certain partial sums involving these excursions. The limits in these laws are related to the volume asymptotic of the thin parts. By Siegel-Veech theory, these are given by Siegel-Veech constants. As a direct consequence, we show that the word metric of mapping classes that approximate a Teichmüller geodesic ray that is random with respect to the Masur-Veech measure, grows faster than $T \log T$.
\end{abstract}

\section{INTRODUCTION}

This paper provides a specific analogy between non-uniform lattices in $S L(2, \mathbb{R})$ and mapping class groups. The analogy is established from the point of view of cusp excursions of random geodesics. For non-uniform lattices in $S L(2, \mathbb{R})$, we consider excursions of a random geodesic in horoball neighborhoods of the cusps of the quotient hyperbolic surface. For a $S L(2, \mathbb{R})$ orbit closure in a stratum of a moduli space of quadratic differentials we consider excursions of random Teichmüller geodesics in a thin part of the orbit closure.

1.1. Non-uniform lattices in $S L(2, \mathbb{R})$. Let $\Gamma$ be a non-uniform lattice in $S L(2, \mathbb{R})$. The quotient $X=\Gamma \backslash \mathbb{H}^{2}$ is a complete finite area surface/orbifold with finitely many cusps $c_{1}, \ldots, c_{J}$. Let $X_{\text {cusps }} \subset X$ be the union of disjoint horoball neighborhoods of the cusps. The set $X_{\text {cusps }}$ lifts to a countable collection $\mathcal{H}$ of disjoint horoballs in $\mathbb{H}^{2}$. The complement $X \backslash X_{\text {cusps }}$ is a compact set $X_{\text {thick }}$ called the thick part of $X$. The lift $\widetilde{X}_{\text {thick }}$ which we call the thick part in $\mathbb{H}^{2}$, is the complement of the union of horoballs in $\mathcal{H}$.

The Liouville measure is a natural $S L(2, \mathbb{R})$-invariant measure on the unit tangent bundle $T^{1} \mathbb{H}^{2}$. In the upper half-plane model it is given by

$$
d \ell=\frac{d x d y d \theta}{2 \pi y^{2}}
$$

The hyperbolic geodesic flow on $T^{1} X$ is given by the action of the diagonal subgroup. The measure $d \ell$ descends to a flow invariant measure on $T^{1} X$. We will continue to call it Liouville measure. The conditional measure on the unit tangent circle at any point is the pullback via the visual map of the standard Lebesgue measure on $\partial \mathrm{H}^{2}=S^{1}$.

2010 Mathematics Subject Classification. 30F60, 32G15.

Key words and phrases. Teichmüller theory, Moduli of Riemann surfaces. 
Since the number of horoballs is countable, $d \ell$-almost every geodesic ray is recurrent to $X_{\text {thick }}$. By the ergodicity of the geodesic flow and the fellow traveling property of hyperbolic geodesics, Leb-almost every geodesic ray from a fixed base-point $x_{0} \in X$ ventures into $X_{\text {cusps }}$ infinitely often. In particular, a geodesic ray $\gamma$ in $\mathbb{H}^{2}$ whose endpoint $r$ in $S^{1}$ is Leb-typical enters and leaves infinitely many horoballs. By analyzing the collection $\mathcal{H}$, Sullivan [28] showed that the lim sup of the maximum depth that $\gamma$ achieves in $X_{\text {cusps }}$ is asymptotically $(1 / 2) \log T$, where $T$ is the time along $\gamma$. It is convenient to assume $x_{0} \in X_{\text {thick }}$ which can be achieved by making $X_{\text {cusps }}$ smaller, if necessary.

To set up notation, let $\gamma\left(x_{0}, r\right)$ be the geodesic ray from $x_{0}$ to $r \in S^{1}$. We denote by $\gamma_{T}\left(x_{0}, r\right)$ the point on $\gamma$ distance $T$ from $x_{0}$. When the context is clear we will use just $\gamma$ and $\gamma_{T}$. Let $\pi: \mathbb{H}^{2} \rightarrow \widetilde{X}_{\text {thick }}$ be the closest point projection. Let $N=N(T)$ be the number of horoballs that $\gamma$ intersects up to $\gamma_{T}$. We enumerate this collection of horoballs $\mathcal{H}(\gamma, T)=\left\{H_{1}, H_{2}, \ldots, H_{N}\right\}$ in the order of increasing time. For all $k<N, \gamma$ enters and exits $H_{k} ; H_{N}$ may be an exception if

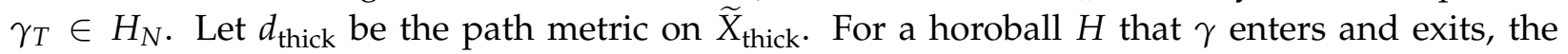
complete excursion $E(\gamma, H)$ is defined as the $d_{\text {thick }}$-distance between the entry and exit points. If $\gamma_{T} \in H_{N}$ then the partial excursion $E\left(\gamma, H_{N}\right)$ is the $d_{\text {thick }}$-distance between the entry point for $H_{N}$ and $\pi\left(\gamma_{T}\right)$.

The total excursion $E(\gamma, T)$ till time $T$, defined as in [15], is

$$
E(\gamma, T)=\sum_{k \leqslant N} E\left(\gamma, H_{k}\right)
$$

Along Leb-typical geodesic rays, [15, Proposition 5.4] shows that $E(\gamma, T) / T \rightarrow \infty$. We prove here:

Theorem 1.2. For Lebesgue almost every $r$ in $S^{1}$,

$$
\lim _{T \rightarrow \infty} \frac{E(\gamma, T)-\max _{k \leqslant N} E\left(\gamma, H_{k}\right)}{T \log T}=\left(\frac{2}{\pi}\right) \frac{\ell\left(T^{1} X_{\text {cusp }}\right)}{\ell\left(T^{1} X\right)} .
$$

1.2.1. Continued fractions. Let $r \in[0,1]$. The continued fraction expansion is given by writing $r$ as

$$
r=\frac{1}{a_{1}+\frac{1}{a_{2}+\frac{1}{+\cdots}}}
$$

where each $a_{i} \in \mathbb{N}$. When $r$ is irrational the expansion is infinite. We denote $r$ as $\left[a_{1}, a_{2}, \ldots\right]$.

Theorem 1.3 (Diamond-Vaaler [9]). For Leb-almost every $r \in[0,1]$

$$
\lim _{n \rightarrow \infty} \frac{\sum_{i=1}^{n} a_{i}-\max _{i \leqslant n} a_{i}}{n \log n}=\frac{1}{\log 2} .
$$

We will derive Theorem 1.3 from Theorem 1.2 in the special case when $X$ is the modular surface $S L(2, \mathbb{Z}) \backslash \mathbb{H}^{2}$. Excursions of geodesic rays into the maximal cusp neighborhood in $X$ are related to coefficients in the continued fraction expansion of the point at infinity. Diamond-Vaaler used techniques specific to the symbolic dynamics (Gauss map) in the theory of continued fractions. Theorem 1.2 relies on more general features viz. asymptotic for $\ell\left(X_{\text {cusps }}\right)$ and exponential mixing of the geodesic flow. These features hold for the Teichmüller geodesic flow in the analogous setting of quadratic differentials. Hence, Theorem 1.9. 
1.3.2. Word metric along random geodesics. As a direct implication of Theorem 1.2, we analyze how the word metric grows along random geodesics.

For distinct points $x, y \in \mathbb{H}^{2}$ let $\gamma(x, y)$ be the hyperbolic geodesic between them. The projected path is defined by $p(x, y)=\pi(\gamma(x, y))$. Let $L(x, y)$ be the $d_{\text {thick }}$-length of $p(x, y)$, where $d_{\text {thick }}$ is the path metric on the thick part. The quantity $L\left(x_{0}, \gamma_{T}\right)-E(\gamma, T)$ is the time spent by $\gamma$ in the interior of $X_{\text {thick. }}$. By the ergodicity of geodesic flow, $L\left(x_{0}, \gamma_{T}\right)-E(\gamma, T)$ grows linearly in $T$. Hence, a direct consequence of Theorem 1.2 is:

Theorem 1.4. For Leb-almost every $r \in S^{1}$

$$
\lim _{T \rightarrow \infty} \frac{L\left(x_{0}, \gamma_{T}\right)-\max _{k \leqslant N} E\left(\gamma, H_{k}\right)}{T \log T}=\left(\frac{2}{\pi}\right) \frac{\ell\left(T^{1} X_{\text {cusp }}\right)}{\ell\left(T^{1} X\right)} .
$$

By [15, Lemma 5.1], the projected path $p\left(x_{0}, \gamma_{T}\right)$ is a quasi-geodesic in $\left(\widetilde{X}_{\text {thick }}, d_{\text {thick }}\right)$. A closer look at the proof of [15, Lemma 5.1] shows that $L\left(x_{0}, \gamma_{T}\right)-d_{\text {thick }}\left(x_{0}, \gamma_{T}\right)$ grows at most linearly in $N$. In Lemma 3.8, we show that $N$ grows linearly in $T$. As a direct consequence of these linear bounds and Theorem 1.4 we get:

Theorem 1.5. For Leb-almost every $r \in S^{1}$

$$
\lim _{T \rightarrow \infty} \frac{d_{\text {thick }}\left(x_{0}, \gamma_{T}\right)-\max _{k \leqslant N} E\left(\gamma, H_{k}\right)}{T \log T}=\left(\frac{2}{\pi}\right) \frac{\ell\left(T^{1} X_{\text {cusp }}\right)}{\ell\left(T^{1} X\right)} .
$$

A base-point $x_{0} \in \mathbb{H}^{2}$ is said to be generic if the stabilizer of $x_{0}$ in $\Gamma$ is trivial. The $\Gamma$-orbit of $x_{0}$ is called a lattice. When $x_{0}$ is generic each lattice point corresponds to a unique group element. Each point $\gamma_{T}$ has at least one closest lattice point which we denote by $h_{T} x_{0}$. In fact, this closest point is unique for almost all points along $\gamma$.

The group $\Gamma$ is finitely generated. A finite choice of generators for $\Gamma$ defines a proper word metric $d_{\Gamma}$ on $\Gamma$. Different choices of generators produce quasi-isometric metrics. Let $d_{\Gamma}\left(1, h_{T}\right)$ be the word length where $h_{T} x_{0}$ is the lattice point closest to $\gamma_{T}$.

The group $\Gamma$ acts cocompactly on $\widetilde{X}_{\text {thick }}$. So by the $\check{S}_{\text {varc-Milnor lemma, }}\left(\Gamma, d_{\Gamma}\right)$ is quasi-isometric to $\left(\widetilde{X}_{\text {thick }}, d_{\text {thick }}\right)$. Thus, as a direct consequence of Theorem 1.5 we get:

Theorem 1.6. There exists a constant $M_{1}>0$ that depends on the word metric such that for Leb-almost every $r \in S^{1}$

$$
M_{1} T \log T<d_{\Gamma}\left(1, h_{T}\right)
$$

for $T$ sufficiently large depending on $r$.

In fact, if the contribution from the largest excursion is removed, then the word metric grows like $T \log T$ up to uniform multiplicative and additive constants. Theorem 1.6 should be thought of as a refinement of Proposition 5.6 in [15] which states that along a Leb-generic geodesic ray the ratio $d_{\Gamma}\left(1, h_{T}\right) / T \rightarrow \infty$ as $T \rightarrow \infty$.

1.7. Moduli spaces of quadratic differentials. Let $S$ be a hyperbolic surface of finite type. $S$ is non-sporadic if it is not a sphere with at most four punctures or boundary components, or a torus with at most one puncture or boundary component. In the sporadic examples, the Teichmüller space is either trivial or isometric to $\mathbb{H}^{2}$ and the mapping class group is a non-uniform lattice in $S L(2, \mathbb{R})$. This reduces us to the previous case.

The Teichmüller space $\mathcal{T}(S)$ is the space of marked conformal structures on $S$, or alternatively, marked hyperbolic metrics on $S$. The mapping class group $\operatorname{Mod}(S)$ is the group of orientation preserving diffeomorphisms of $S$ modulo isotopy. $\operatorname{Mod}(S)$ acts on $\mathcal{T}(S)$ by changing the marking. The quotient $\mathcal{M}(S)=\operatorname{Mod}(S) \backslash \mathcal{T}(S)$ is the moduli space of Riemann surfaces. 
The Teichmüller metric is given by

$$
d_{\mathcal{T}}(X, Y)=\frac{1}{2} \inf _{f} \log K(f),
$$

where the infimum is over all quasi-conformal maps $f: X \rightarrow Y$, and $K(f)$ is the quasi-conformal constant of $f$. The group $\operatorname{Mod}(S)$ acts by isometries of $d_{\mathcal{T}}$. Thus, the metric descends to a metric on $\mathcal{M}(S)$. The thin part of $\mathcal{T}(S)$ consists of hyperbolic surfaces that contain a simple closed curve with small hyperbolic length. The thin part of $\mathcal{T}(S)$ is obviously $\operatorname{Mod}(S)$ invariant. The quotient $\operatorname{Mod}(S) \backslash \mathcal{T}(S) \subset \mathcal{M}(S)$ is the thin part of the moduli space of Riemann surfaces.

For a Riemann surface $X$, let $\mathcal{Q}(X)$ be the set of meromorphic quadratic differentials on $X$ with simple poles at the punctures. If $\left(k_{1}, k_{2}, \ldots, k_{r}\right)$ are the multiplicities of the zeros then $k_{1}+k_{2}+$ $\cdots+k_{r}=2 g-2+n$, where $n$ is the number of punctures. A quadratic differential is equivalent to a half-translation structure on $S$, i.e. it defines charts from $S$ to $\mathbb{C}$ with transition functions of the form $\pm z+c$. The resulting flat metric has a cone singularity with angle $(k+2) \pi$ at a $k$-order zero (or with $k=-1$ for a simple pole). A quadratic differential is unit area if the corresponding singular flat metric has area 1 . The space of unit area quadratic differentials $\mathcal{Q}$ can be identified with the unit cotangent bundle to $\mathcal{T}(S)$ [16]. The space $\mathcal{Q}$ is stratified by the multiplicity of its zeros: we denote the stratum with multiplicities $\alpha=\left(k_{1}, k_{2}, \ldots, k_{r}\right)$ by $\mathcal{Q}(\alpha)$. Each stratum is $\operatorname{Mod}(S)$ invariant. We will continue to denote the moduli space by $\mathcal{Q}(\alpha)$.

The periods/holonomies of a fixed basis for the homology of $S$ relative to the singularities, give local co-ordinates on $\mathcal{Q}(\alpha)$. The natural volume form $\mu_{\text {hol }}$ in these co-ordinates, called the MasurVeech measure, can be thought of as an analog of the Liouville measure. It is invariant under $\operatorname{Mod}(S)$-action and descends to a finite measure on the moduli space. See [22], [29].

The affine action of $S L(2, \mathbb{R})$ on $\mathbb{C}=\mathbb{R}^{2}$ preserves the transitions $z \rightarrow \pm z+c$. This defines $S L(2, R)$ action on each stratum $\mathcal{Q}(\alpha)$. The action of the diagonal part defines the Teichmüller geodesic flow. The compact part $S O(2, \mathbb{R})$ leaves the underlying conformal structure unchanged. This gives an isometric embedding $\mathbb{H}^{2}=S L(2, \mathbb{R}) / S O(2, R) \rightarrow \mathcal{T}(S)$. These are called Teichmüller discs and they foliate $\mathcal{T}(S)$. We denote by $S L(2, \mathbb{R})(q)$ the orbit and by $\mathbb{D}(q)$ the Teichmüller disc.

In the flat metric defined by $q$, a saddle connection is a geodesic segment in the $q$-metric that connects a pair of (same or distinct) singularities. For $\epsilon>0$, the $\epsilon$-thin part $\mathcal{Q}(\alpha)_{\epsilon}$ is the set of $q \in \mathcal{Q}(\alpha)$ such that some saddle connection has $q$-length squared less than $\epsilon$.

The points $q^{\prime}$ in $S L(2, \mathbb{R})(q)$ for which a particular saddle connection $\beta$ has $q^{\prime}$-length squared shorter than $\epsilon$ projects to a horoball $H$ in $\mathbb{D}(q)$. The point at infinity for $H$ is given by the direction in which $\beta$ is vertical. For saddle connections that are parallel for $q$, the ratios of their holonomies are constant on $S L(2, \mathbb{R})(q)$. Thus, in a collection of parallel saddle connections, the shortest holonomy will be used to define the horoball.

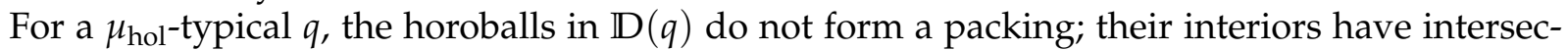
tions. Every point $X \in \mathbb{D}(q)$ is contained in at most finitely many horoballs. But there need not be an upper bound over $\mathbb{D}(q)$ for this number. This makes it hard to estimate the $d_{\text {thick }}$-distance between the entry and exit points of a geodesic in $\mathcal{Q}(\alpha)_{\epsilon}$, which would have been a natural analog for an excursion in this context. Instead, we consider excursions in individual horoballs.

The excursions in horoballs in $\mathbb{D}(q)$ of a geodesic $\gamma$ till time $T$ are defined as follows. For a horoball $H$ that $\gamma$ enters and exits, the complete excursion $E(\gamma, H)$ is defined as the distance along $\partial H$ between the entry and exit points. If $\gamma_{T} \in H$ then the partial excursion $E(\gamma, H)$ is defined as the distance along $\partial H$ between the entry point of $\gamma$ and $\pi_{H}\left(\gamma_{T}\right)$ where $\pi_{H}: H \rightarrow \partial H$ is the closest point projection. Let $H_{1}, \ldots, H_{N(T)}$ denote all the horoballs that $\gamma$ intersects till time $T$. The total excursion $E(\gamma, T)$ is defined as

$$
E(\gamma, T)=\sum_{k \leqslant N(T)} E\left(\gamma, H_{k}\right)
$$


When horoballs have disjoint interiors these definitions coincide with the definitions in Section 1.1. With intersections, the excursions $E\left(\gamma, H_{k}\right)$ can be simultaneous and there can be several partial excursions when $\gamma_{T}$ is in the thin part.

The main theorem we prove is:

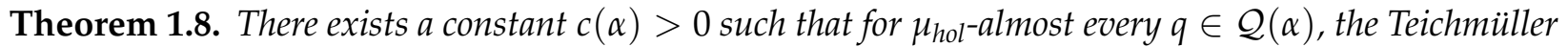
geodesic ray $\gamma$ that $q$ determines satisfies

$$
\lim _{T \rightarrow \infty} \frac{E(\gamma, T)-\max _{k \leqslant N(T)} E\left(\gamma, H_{k}\right)}{T \log T}=2 \epsilon c(\alpha) .
$$

The constant $c(\alpha)$ is the Siegel-Veech constant associated to $\mathcal{Q}(\alpha)$. See Section 4.5 for the definition of Siegel-Veech constants.

Theorem 1.8 is a special case of a more general theorem viz. Theorem 1.9 for $S L(2, \mathbb{R})$-invariant measures and $S L(2, \mathbb{R})$ invariant loci of saddle connection holonomies.

$S L(2, \mathbb{R})$ orbit closures and invariant measures. Recently, Eskin and Mirzakhani [12, Theorem 1.4] showed that ergodic $S L(2, \mathbb{R})$-invariant probability measures are of Lebesgue class and supported on invariant complex submanifolds in $\mathcal{Q}(\alpha)$. These manifolds are affine in the sense that they are given by linear equations in the holonomy co-ordinates. Furthermore, Eskin, Mirzakhani and Mohammadi [13, Theorem 2.1] showed that all $S L(2, \mathbb{R})$ orbit closures are affine invariant submanifolds. See [12, Section 1] for more details. More recently, Filip showed that these submanifolds are algebraic subvarieties. [14].

Let $\mu$ be an ergodic $S L(2, \mathbb{R})$-invariant probability measure supported on an affine invariant submanifold $\mathcal{N}$. For $\epsilon>0$, the $\epsilon$-thin part $\mathcal{N}_{\epsilon}$ is the subset of $q \in \mathcal{N}$ such that some saddle connection has $q$-length squared less than $\epsilon$. Saddle connections $\beta_{1}, \beta_{2}$ are $\mathcal{N}$-parallel if they are parallel for an open set of quadratic differentials in $\mathcal{N}$. We assume that $\mu$ satisfies the following regularity condition. For $\epsilon, \mathcal{\kappa}>0$, let $\mathcal{N}_{\epsilon, \kappa}$ be the subset of $q \in \mathcal{N}$ that have at least one saddle connection $\beta_{1}$ with $\ell_{q}^{2}\left(\beta_{1}\right) \leqslant \epsilon$ and a saddle connection $\beta_{2}$ not $\mathcal{N}$-parallel to $\beta_{1}$ with $\ell_{q}^{2}\left(\beta_{2}\right) \leqslant \kappa$. We assume that there exists $m_{1}>0$ such that for $\epsilon, \kappa$ small enough

$$
\mu\left(\mathcal{N}_{\epsilon, \kappa}\right) \leqslant m_{1} \epsilon \kappa
$$

For $\mu_{\text {hol }},[23$, Section 10, Claim (7)] proves the regularity above. A weaker regularity for any $S L(2, \mathbb{R})$-invariant measure is proved in [4, Theorem 1.2].

$S L(2, \mathbb{R})$-invariant loci. For $q \in \mathcal{N}$, let $V(q) \in \mathbb{R}^{2} \backslash\{(0,0)\}$ be an assignment of a non-empty subset (with multiplicity) of holonomies of saddle connections on $q$. We require that the assignment varies linearly under $S L(2, \mathbb{R})$ action, i.e. $V(g q)=g V(q)$ for all $g \in S L(2, \mathbb{R})$. Such an assignment $V$ will be called a $S L(2, \mathbb{R})$-invariant locus. Let $c(V, \mu)$ be the Siegel-Veech constant associated to $V$ and $\mu$. See Section 4.5 for the definition. We assume $V$ satisfies $c(V, \mu)>0$.

For $R \geqslant 1$, the $\epsilon / R$-thin part of $\mathcal{N}$ corresponding to $V$ is the subset of $q \in \mathcal{N}$ for which some saddle connection with holonomy in $V(q)$ has $q$-length squared less than $\epsilon / R$. We denote the set by $\mathcal{N}(V)_{\epsilon / R}$. The regularity condition and the Siegel-Veech formula 4.6 can be used to prove the volume asymptotic

$$
\lim _{R \rightarrow \infty} \frac{\mu\left(\mathcal{N}(V)_{\epsilon / R}\right)}{\pi \epsilon / R}=c(V, \mu) .
$$

See [11, Section 7] for the main ideas.

Here, for $\mu$-almost every $q \in \mathcal{N}$, we consider excursions of the Teichmüller geodesic ray determined by $q$ in horoballs in $\mathbb{D}(q)$ for saddle connections with holonomy in $V$. Let $E_{V}(\gamma, T)$ be the sum till time $T$ of excursions of $\gamma$ in horoballs for saddle connections with holonomy in $V$. Let $N_{V}$ be the number of such excursions of $\gamma$ till time $T$. The main theorem we prove is the following: 
Theorem 1.9. Let $\mu$-be a regular $S L(2, \mathbb{R})$-invariant measure supported on an affine invariant submanifold $\mathcal{N}$. For $\mu$-almost every $q \in \mathcal{N}$, the Teichmüller geodesic ray $\gamma$ that $q$ determines satisfies

$$
\lim _{T \rightarrow \infty} \frac{E_{V}(\gamma, T)-\max _{k \leqslant N_{V}} E\left(\gamma, H_{k}\right)}{T \log T}=2 \epsilon c(V, \mu),
$$

where $c(V, \mu)$ is the Siegel-Veech associated to $V$ and $\mu$.

Theorem 1.8 is a special case of Theorem 1.9 when $\mathcal{N}=\mathcal{Q}(\alpha), \mu=\mu_{\text {hol }}$ and $V$ is the $S L(2, \mathbb{R})$ invariant locus of holonomies of all saddle connections.

1.9.1. Configurations with cylinders. For the analog of Theorem 1.6, we state a special case of Theorem 1.9. For completeness we give some background.

For a connected component of $\mathcal{Q}(\alpha)$, a configuration $\mathcal{C}$ of saddle connections is a geometric type of maximal collections of homologous saddle connections on a translation or half-translation surface in it. Here, the homology is the appropriate relative homology; see [24] for details. The condition in homology implies that the saddle connections in a configuration are parallel. For holomorphic 1-forms their holonomies coincide. For quadratic differentials their holonomies can take two values which differ by a factor of 2 . The saddle connections with the smaller holonomy will be called the small saddle connections in $\mathcal{C}$. It was shown in [11] and [24] that in a $\mu_{\text {hol-typical }}$ degeneration all saddle connections in some configuration shrink to length zero. A configuration $\mathcal{C}$ gives a $S L(2, \mathbb{R})$-invariant locus $V_{\mathcal{C}}$ for $\mathcal{Q}(\alpha)$. It follows that $c\left(V, \mu_{\text {hol }}\right)>0$.

A special subset of configurations corresponds to metric cylinders. A metric cylinder is an embedded cylinder that is a union of freely homotopic closed trajectories of $q$ such that the boundary components are a concatenation of saddle connections. If some of the saddle connections in a configuration $\mathcal{C}$ bound a metric cylinder, we call $\mathcal{C}$ a configuration with cylinders. Masur and Zorich [24] show that each boundary component of such cylinders has exactly one or two saddle connections in $\mathcal{C}$. The $q$-length of the core curve is equal to the boundary saddle connection or twice the length of one of the boundary saddle connections depending on the case.

Given $\mathcal{C}$, the $\mathcal{C}$-thin part of $S L(2, \mathbb{R})(q)$ are points for which the length squared of the small saddle connections in $\mathcal{C}$ is less than $\epsilon$. Its projection to $\mathbb{D}(q)$ is a horoball. The point at infinity for the horoball is the direction in which the saddle connections in $\mathcal{C}$ are vertical.

If $\epsilon$ is sufficiently small compared to the $q$-area of a cylinder then the core curve is also short in the hyperbolic metric. For $0<\sigma<1$ small enough depending on the orbit closure, we can specialize further to configurations with cylinders in which some cylinder has area at least $\sigma$. Such a restriction gives a horoball "packing": any point in $\mathbb{D}(q)$ is contained at most $3 g-3+n$ horoballs. By construction, the packing is $\operatorname{Mod}(S)$ equivariant. Masur [22] showed that in each Teichmüller disc the packing satisfies Sullivan's criteria and used it to prove the lower bound in the log law: a Leb-typical geodesic ray in every Teichmüller disc is recurrent to the thick part with $\lim$ sup of the maximum depth in $\mathcal{T}(S)_{\epsilon}$ asymptotically of size $(1 / 2) \log T$.

Let $V$ be the subset of holonomies of saddle connections forming configurations with cylinders such that some cylinder has area at least $\sigma$. It follows from the Siegel-Veech machinery and [22, Proposition 2.5] that if $\sigma$ is small enough depending on $\mathcal{Q}(\alpha)$ then $c_{\mathrm{cyl}}>0$, where $c_{\mathrm{cyl}}{ }_{\sigma}$ is the Siegel-Veech constant for $\mathcal{Q}(\alpha)$ for configurations with cylinders such that some cylinder is of area at least $\sigma$. For translation surfaces, there are finer results due to Vorobets. For example, see [30, Theorem 1.8]. For a geodesic $\gamma \in \mathcal{Q}(\alpha)$, let $E_{\mathrm{cyl}_{\sigma}}(\gamma, T)$ be the sum till time $T$ of excursions of $\gamma$ in horoballs for such configurations. Let $N_{\mathrm{cyl}}(T)$ be the number of such excursions till time $T$. As a special case of Theorem 1.9 we have 


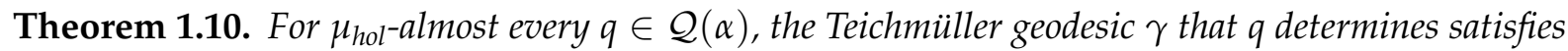

$$
\lim _{T \rightarrow \infty} \frac{E_{c y l_{\sigma}}(\gamma, T)-\max _{k \leqslant N_{c y l_{\sigma}}} E\left(\gamma, H_{k}\right)}{T \log T}=2 \epsilon c_{c y l_{\sigma}}(\alpha),
$$

where $c_{c y l}(\alpha)$ is the Seigel-Veech constant for $\mathcal{Q}(\alpha)$ for configurations with cylinders such that some cylinder is of area at least $\sigma$.

Using Theorem 1.10 for the principal stratum, we can prove a lower bound on word-metric growth along typical Teichmüller geodesics. Along a Teichmüller geodesic, the twisting in the core curve of a metric cylinder is up to a uniform multiplicative constant, $A / \epsilon$ times the excursion, where $A$ is the $q$-area of the cylinder. See [15, Proposition 2.7]. By Mumford compactness, the closure of $\mathcal{M}(S) \backslash \mathcal{M}(S)_{\epsilon}$ is compact. Hence, by Švarc-Milnor lemma, $\operatorname{Mod}(S)$ is quasi-isometric to the thick part $\mathcal{T}(S) \backslash \mathcal{T}(S)_{\epsilon}$. With a base-point $X_{0}$ in the thick part, the orbit $\operatorname{Mod}(S) X_{0}$ will be called a Teichmüller lattice. If $\gamma$ is recurrent to the thick part then along recurrence times $\gamma_{T}$, there is a lattice point $h_{T} X_{0}$ closes to $\gamma_{T}$. The distance between $\gamma_{T}$ and $h_{T} X_{0}$ is bounded by the diameter of $\mathcal{M}(S) \backslash \mathcal{M}(S)_{\epsilon}$. Because of compactness, this diameter is finite. As shown in [15, Proposition 3.11], along a recurrent Teichmüller geodesic $\gamma$ the total excursion $E(\gamma, T)$ in the Masur collection gives a coarse lower bound on the word metric of the approximating group elements $h_{T}$, i.e. there exists constants $a_{1}, a_{2}>0$ such that

$$
d_{\operatorname{Mod}(S)}\left(1, h_{T}\right) \geqslant a_{1} E(\gamma, T)-a_{2} .
$$

Thus, as a direct consequence of Theorem 1.10 in the principle stratum we get

Theorem 1.11. There exists a constant $M_{2}>0$ depending on the word metric such that for almost every $q \in \mathcal{Q}\left(X_{0}\right)$ the approximating group elements $h_{T}$ along the Teichmüller geodesic $\gamma$ that $q$ determines satisfy

$$
M_{2} T \log T<d_{\operatorname{Mod}(S)}\left(1, h_{T}\right)
$$

for all $T$ sufficiently large depending on $q$.

Suppose $q_{1}, q_{2}$ have the same uniquely ergodic measured foliation as their vertical foliation. For such quadratic differentials, Masur [20, Theorem 2] showed then that the geodesic rays that they determine are positively asymptotic. For any base-point $X_{0} \in \mathcal{T}(S)$, a $\mu_{\text {hol }}$-typical $q \in \mathcal{Q}\left(X_{0}\right)$ has a uniquely ergodic vertical foliation. Thus, Theorem 1.11 implies the following theorem.

Theorem 1.12. Let $X_{0} \in \mathcal{T}(S)$ be a base-point. There is a constant $M_{3}>0$ such that for Leb-almost every $q \in Q\left(X_{0}\right)$ the approximating group elements $h_{T}$ along the Teichmüller geodesic $\gamma$ that $q$ determines satisfy

$$
M_{3} T \log T<d_{\operatorname{Mod}(S)}\left(1, h_{T}\right)
$$

for all $T$ sufficiently large depending on $q$.

1.13. Strategy of proof. The main idea is to approximate the sum of excursions till $T$ by an integral over time of a function defined over $T^{1} X_{\text {cusps }}$ or $\mathcal{N}(V)_{\epsilon}$. This function is not $L^{1}$. Analyzing the largest excursion, we prove that if it exceeds $T(\log T)^{c}$ for some $1>c>1 / 2$, then it is the unique excursion that exceeds this threshold. This follows from a Borel-Cantelli argument which requires quasi-independence of excursions. We use mixing of the geodesic flow to establish quasiindependence. See Proposition 3.2. By removing the largest excursion from the sum we get a quantity that can be approximated by a suitable $T$-dependent truncation of the above function. This truncation is $L^{1}$. The leading term of its $L^{1}$ norm is a constant times $\log T$. The constant is in terms of the proportional volume of the cusp neighborhoods. To conclude the main theorems, we apply an effective ergodic theorem to the truncation. This shows that the integral over $[0, T]$ of the truncation is equal to $T$ times the $L^{1}$ norm of the truncation with an error term which is $o(T \log T)$. 
To prove the effective ergodic theorem viz. Theorem 2.4, we use a decay of correlations for the geodesic flow. This decay of correlations is independently due to Moore and Ratner [26] [27] in the context of non-uniform lattices. For quadratic differentials, the decay is due to Avila-Resende [5] for the Masur-Veech measure, and Avila-Gouëzel [2] for general $S L(2, \mathbb{R})$-invariant measures.

In the setting of quadratic differentials matters are complicated by the fact that a half-translation surface can have several non-parallel short saddle connections. While this number is finite for any given half-translation surface there is no upper bound for it over the $S L(2, \mathbb{R})$-orbit closure. This means that a Teichmüller geodesic can do several excursions simultaneously and typically it does so. We impose a regularity condition on the $S L(2, \mathbb{R})$-invariant measure, namely quasiindependence for two non-parallel saddle connections to be simultaneously short. To prove that the truncation is $L^{1}$ the main technical work leverages two facts: the above quasi-independence and a bound due to Eskin and Masur [10] for the number of short saddle connections in terms of length of shortest saddle connection. We also show that the leading term of its $L^{1}$-norm is asymptotically a constant times $\log T$. The constant is related to the asymptotic of volumes of thin parts. By Siegel-Veech theory, these are the associated Siegel-Veech constants.

1.14. Acknowledgements. The central question considered in the paper arose in joint work with J. Maher and G. Tiozzo [15]. I thank them for the initial discussion and for pointing out the paper by Diamond and Vaaler [9]. I thank J. Chaika, C. Matheus and M. Pollicott for useful discussions about the ergodic theory, J. Athreya and A. Eskin for useful discussions about the volume asymptotics in moduli spaces of quadratic differentials. I thank the Institut Henri Poincaré and the Newton Institute for their hospitality. The research was supported by a Global Research Fellowship with the Institute of Advanced Study at the University of Warwick. I thank the referee for comments that improved the paper.

\section{ERGODIC THEORY}

This section develops the ergodic theoretic tools which will be used later. In particular, the main goal is to derive the effective ergodic theorem viz. Theorem 2.4 which gives a uniform rate of convergence in the ergodic theorem simultaneously for a sequence of non-negative functions that satisfy a certain decay of correlations.

Let $(X, \mathcal{B}, \ell)$ be a probability measure space. Let $g_{t}$ be a measure preserving flow on $X$ such that $g_{t}$ is exponentially mixing. More precisely, we assume that an appropriate subspace of $L^{2}(X)$ satisfies following decay of correlations: if $f_{1}$ and $f_{2}$ are functions in the subspace then $\int_{X} f_{1} d \ell=$ $\int_{X} f_{2} d \ell=0$ and there exists constants $K, \rho>0$ such that

$$
\left|\int_{X} f_{1}\left(g_{s} x\right) f_{2}\left(g_{t} x\right) d \ell\right| \leqslant K|t-s| e^{-\rho|t-s|}\left\|f_{1}\right\|_{L^{2}}\left\|f_{2}\right\|_{L^{2}} .
$$

For a function $f \in L^{1}(X)$, let $I(f)=\int_{X} f d \ell$. We denote by $\mathcal{L}$ the subspace in $L^{2}(X)$ of functions $f$ such that the function $f-I(f)$ satisfies the decay of correlations 2.1 above.

Lemma 2.2. Any function $f \in \mathcal{L}$ with $I(f)=0$ satisfies:

$$
\int_{X}\left(\int_{0}^{T} f\left(g_{t} x\right) d t\right)^{2} d \ell \leqslant 2 K T\|f\|_{L^{2}}^{2} .
$$


Proof. Observe that

$$
\begin{aligned}
\int_{X}\left(\int_{0}^{T} f\left(g_{t} x\right) d t\right)^{2} d \ell & =\int_{X}\left(\int_{0}^{T} \int_{0}^{T} f\left(g_{s} x\right) f\left(g_{t} x\right) d s d t\right) d \ell \\
& =\int_{0}^{T} \int_{0}^{T}\left(\int_{X} f\left(g_{s} x\right) f\left(g_{t} x\right) d \ell\right) d s d t \\
& \leqslant \int_{0}^{T} \int_{0}^{T} K|t-s| e^{-\rho|t-s|}\|f\|_{L^{2}}^{2} d s d t
\end{aligned}
$$

where we have used the decay of correlations 2.1 in the last inequality. A direct computation shows

$$
\begin{aligned}
\int_{0}^{T} \int_{0}^{T} K|t-s| e^{-\rho|t-s|}\|f\|_{L^{2}}^{2} d s d t & =K\|f\|_{L^{2}}^{2}\left(\frac{T}{\rho^{2}}\left(1+e^{-\rho T}\right)+\frac{2}{\rho^{3}}\left(-1+e^{-\rho T}\right)\right) \\
& \leqslant 2 K T\|f\|_{L^{2}}^{2}
\end{aligned}
$$

finishing the proof of the lemma.

Suppose $n: \mathbb{R} \rightarrow \mathbb{N}$ is a function that is constant on each interval $\left[2^{k}, 2^{k+1}\right)$.

Theorem 2.4. For any $c>1 / 2, m>1$ and any sequence of non-negative functions $f_{j} \in \mathcal{L}$, almost every $x$ satisfies

$$
\begin{aligned}
\frac{1}{m} T\left\|f_{n}\right\|_{L^{1}}-T^{1 / 2}(\log T)^{c}\left(\left\|f_{n}\right\|_{L^{2}}^{2}-\left\|f_{n}\right\|_{L^{1}}^{2}\right)^{1 / 2} & \leqslant \int_{0}^{T} f_{n}\left(g_{t} x\right) d t \\
& \leqslant m T\left\|f_{n}\right\|_{L^{1}}+T^{1 / 2}(\log T)^{c}\left(\left\|f_{n}\right\|_{L^{2}}^{2}-\left\|f_{n}\right\|_{L^{1}}^{2}\right)^{1 / 2}
\end{aligned}
$$

for all $T$ large enough depending on $x$ and $m$ and where $n=n(T)$.

Proof. Given a function $f \in \mathcal{L}$ define

$$
F(x)=f(x)-I(f) .
$$

Then $I(F)=0$ and so by Lemma 2.2

$$
\int_{X}\left(\int_{0}^{T} F\left(g_{t} x\right) d t\right)^{2} d \ell \leqslant 2 K T\|F\|_{L^{2}}^{2}
$$

for all $T$. By Chebysheff's inequality, for any positive function $r(T, F)$ we have

$$
\ell\left(x \text { such that }\left(\int_{0}^{T} F\left(g_{t} x\right) d t\right)^{2} \geqslant r(T, F)\right) \leqslant \frac{2 K T\|F\|_{L^{2}}^{2}}{r(T, F)} .
$$

Let $c>1 / 2$ and set $r(T, F)=T(\log T)^{2 c}\|F\|_{L^{2}}^{2}$ in 2.5. Then we get

$$
\ell\left(x \text { such that }\left(\int_{0}^{T} F\left(g_{t} x\right) d t\right)^{2} \geqslant T(\log T)^{2 c}\|F\|_{L^{2}}^{2}\right) \leqslant \frac{2 K}{(\log T)^{2 c}} .
$$

Starting from our sequence $f_{j}$, let $F_{j}$ be the sequence of functions given by

$$
F_{j}(x)=f_{j}(x)-I\left(f_{j}\right) .
$$

The estimate 2.6 above is satisfied by all functions $F_{j}$ and in particular by $F_{n}$ where $n=n(T)$. Fix $r=1 / a$ for some positive integer $a>1$. Observe that for the sequence $T_{k}=2^{r k}$

$$
\sum_{k=1}^{\infty} \frac{2 K}{\left(\log T_{k}\right)^{2 c}}=\sum_{k=1}^{\infty} \frac{2 K}{(r k)^{2 c}}<\infty
$$


Hence by Borel-Cantelli lemma, almost every $x$ satisfies

$$
\left(\int_{0}^{T_{k}} F_{n}\left(g_{t} x\right) d t\right)^{2} \leqslant T_{k}\left(\log T_{k}\right)^{2 c}\left\|F_{n}\right\|_{L^{2}}^{2}
$$

for all $k$ large enough depending on $x$ and $a$. Similarly, setting $r(T, F)=\left(T / 2^{r}\right)\left(\log \left(T / 2^{r}\right)\right)^{2 c}\|F\|_{L^{2}}^{2}$ and shifting $n(T)$ to $n(T / 2)$, the same reasoning by Borel-Cantellii lemma implies that almost every $x$ satisfies

$$
\left(\int_{0}^{T_{k+1}} F_{n}\left(g_{t} x\right) d t\right)^{2} \leqslant T_{k}\left(\log T_{k}\right)^{2 c}\left\|F_{n}\right\|_{L^{2}}^{2}
$$

for all $k$ large enough depending on $x$ and $a$. Hence a full measure set of $x$ satisfy both 2.7 and 2.8. Noting that $F_{n}\left(g_{t} x\right)=f_{n}\left(g_{t} x\right)-I\left(f_{n}\right)$ and $\left\|F_{n}\right\|_{L^{2}}^{2}=\left\|f_{n}\right\|_{L^{2}}^{2}-I\left(f_{n}\right)^{2}$, the above estimates can be rewritten as

$$
\left|\int_{0}^{T_{k}} f_{n}\left(g_{t} x\right) d t-T_{k} I\left(f_{n}\right)\right| \leqslant T_{k}^{1 / 2}\left(\log T_{k}\right)^{c}\left(\left\|f_{n}\right\|_{L^{2}}-I\left(f_{n}\right)^{2}\right)^{1 / 2}
$$

and

$$
\left|\int_{0}^{T_{k+1}} f_{n}\left(g_{t} x\right) d t-T_{k+1} I\left(f_{n}\right)\right| \leqslant T_{k}^{1 / 2}\left(\log T_{k}\right)^{c}\left(\left\|f_{n}\right\|_{L^{2}}-I\left(f_{n}\right)^{2}\right)^{1 / 2} .
$$

Over the intermediate times $T_{k}<T<T_{k+1}$ the number $n$ does not vary. So the function $f_{n}$ being considered remains the same. Now we use the assumption that $f_{n}$ is a non-negative function to get an estimate such as above for these intermediate times. Since $f_{n}$ is non-negative, the time integral of $f_{n}$ is non-decreasing. In particular,

$$
\int_{0}^{T_{k}} f_{n}\left(g_{t} x\right) d t \leqslant \int_{0}^{T} f_{n}\left(g_{t} x\right) d t \leqslant \int_{0}^{T_{k+1}} f_{n}\left(g_{t} x\right) d t .
$$

Observe that

$$
T_{k} I\left(f_{n}\right)-T_{k}^{1 / 2}\left(\log T_{k}\right)^{c}\left(\left\|f_{n}\right\|_{L^{2}}^{2}-I\left(f_{n}\right)^{2}\right)^{1 / 2} \geqslant \frac{1}{2^{r}} T I\left(f_{n}\right)-T^{1 / 2}(\log T)^{c}\left(\left\|f_{n}\right\|_{L^{2}}^{2}-I\left(f_{n}\right)\right)^{1 / 2}
$$

and

$$
T_{k+1} I\left(f_{n}\right)+T_{k}^{1 / 2}\left(\log T_{k}\right)^{c}\left(\left\|f_{n}\right\|_{L^{2}}^{2}-I\left(f_{n}\right)\right)^{1 / 2} \leqslant 2^{r} T I\left(f_{n}\right)+T^{1 / 2}(\log T)^{c}\left(\left\|f_{n}\right\|_{L^{2}}^{2}-I\left(f_{n}\right)\right)^{1 / 2} .
$$

Finally, note $I\left(f_{n}\right)=\left\|f_{n}\right\|_{L^{1}}$. The left hand side of 2.9 is the lower bound in 2.7 and the left hand side in 2.10 is the upper bound in 2.8. Thus we get

$$
\begin{aligned}
\frac{1}{2^{r}} T I\left(f_{n}\right)-T^{1 / 2}(\log T)^{c}\left(\left\|f_{n}\right\|_{L^{2}}^{2}-I\left(f_{n}\right)\right)^{1 / 2} & \leqslant \int_{0}^{T} f_{n}\left(g_{t} x\right) d t \\
& \leqslant 2^{r} T I\left(f_{n}\right)+T^{1 / 2}(\log T)^{c}\left(\left\|f_{n}\right\|_{L^{2}}^{2}-I\left(f_{n}\right)\right)^{1 / 2} .
\end{aligned}
$$

The theorem follows by choosing $a$ large enough such that $r=1 / a$ satisfies $2^{r}<m$.

We also prove a variant of Lemma 2.2 which we will need later for quasi-independence of excursions.

Lemma 2.11. For any $S_{1}<S_{2}<T$ and non-negative function $f \in \mathcal{L}$

$$
\int_{X}\left(\int_{S_{1}}^{S_{2}} f\left(g_{s} x\right) d s \int_{S_{2}}^{T} f\left(g_{t} x\right) d t\right) d \ell<\left(S_{2}-S_{1}\right)\left(T-S_{2}\right)\|f\|_{L^{1}}^{2}+\frac{5 K}{\rho}\left(\|f\|_{L^{2}}^{2}-I(f)^{2}\right)
$$

where $K, c$ are the constants in the decay of correlations 2.1 . 
Proof. For any function $f \in \mathcal{L}$, define $F$ by

$$
F(x)=f(x)-I(f) .
$$

Then $I(F)=0$ and so it satisfies the decay of correlations 2.1. Note that

$$
\int_{X} F\left(g_{s} x\right) F\left(g_{t} x\right) d \ell=\int_{X} f\left(g_{s} x\right) f\left(g_{t} x\right) d \ell-I(f)^{2}
$$

and $\left\|\left.F\right|_{L^{2}} ^{2}=\right\| f \|_{L^{2}}^{2}-I(f)^{2}$. It follows that $f$ satisfies

$$
\left|\int_{X} f\left(g_{s} x\right) f\left(g_{t} x\right) d \ell-I(f)^{2}\right| \leqslant K|t-s| e^{-\rho|t-s|}\left(\|f\|_{L^{2}}^{2}-I(f)^{2}\right)
$$

which implies

$$
\left|\int_{X} f\left(g_{s} x\right) f\left(g_{t} x\right) d \ell\right| \leqslant I(f)^{2}+K|t-s| e^{-\rho|t-s|}\left(\|f\|_{L^{2}}^{2}-I(f)^{2}\right) .
$$

For non-negative functions this implies

$$
\begin{aligned}
\int_{X}\left(\int_{S_{1}}^{S_{2}} f\left(g_{s} x\right) d s \int_{S_{2}}^{T} f\left(g_{t} x\right) d t\right) d \ell & =\int_{S_{2}}^{T} \int_{S_{1}}^{S_{2}}\left(\int_{X} f\left(g_{s} x\right) f\left(g_{t} x\right) d \ell\right) d s d t \\
& \leqslant \int_{S_{2}}^{T} \int_{S_{1}}^{S_{2}}\left[I(f)^{2}+K|t-s| e^{-\rho|t-s|}\left(\|f\|_{L^{2}}^{2}-I(f)^{2}\right)\right] d s d t \\
& <\left(S_{2}-S_{1}\right)\left(T-S_{2}\right) I(f)^{2}+\frac{5 K}{\rho}\left(\|f\|_{L^{2}}^{2}-I(f)^{2}\right)
\end{aligned}
$$

where the last inequality follows from a direct computation.

\section{PARTIAl SUMS Of EXCURSIONS FOR NON-UNIFORM LATTICES IN $S L(2, \mathbb{R})$}

The Liouville measure $\ell$ on $T^{1} \mathbb{H}^{2}$ is invariant under the $S L(2, \mathbb{R})$ action and descends to a flowinvariant measure on $T^{1} X=\Gamma \backslash T^{1} \mathbb{H}^{2}$. To get a probability measure $\ell$ on $T^{1} X$ we normalize by passing to

$$
d \ell \rightarrow \frac{1}{2 \pi|\chi(X)|} d \ell
$$

For notational simplicity we continue to call the probability measure $d \ell$.

The geodesic flow on $T^{1} X$ is given by the action of the diagonal subgroup of $S L(2, \mathbb{R})$. It is ergodic with respect to $\ell$. In fact, it is known to be exponentially mixing. As shown in [26], $S O(2, \mathbb{R})$-invariant $L^{2}$-functions on $T^{1} X$ satisfy the following decay of correlations for the diagonal flow: There exists constants $K>0, \rho>0$ such that for any pair $f_{1}, f_{2}$ of $S O(2, \mathbb{R})$-invariant $L^{2}$ functions on $T^{1} X$ with $\int_{T^{1} X} f_{1} d \ell=\int_{T^{1} X} f_{2} d \ell=0$

$$
\int_{T^{1} X} f_{1}(x) f_{2}\left(g_{t} x\right) d \ell \leqslant K t e^{-\rho t}\left\|f_{1}\right\|_{L^{2}}\left\|f_{2}\right\|_{L^{2}}
$$

See also [27, Theorem 2], [25, Corollary 2.1]. In particular, the lifts to $T^{1} X$ of $L^{2}$-functions on $X$ are by default $S O(2, \mathbb{R})$ invariant. So the above decay of correlations applies to them.

For $R \geqslant 1$, let $Y_{R}$ be the subset of the horoballs $\mathcal{H}$ consisting of those points which are at least distance $\log R$ from the boundary of the horoballs in the hyperbolic metric, i.e.

$$
Y_{R}:=\bigcup_{H \in \mathcal{H}}\{x \in H: d(x, \partial H) \geqslant \log R\} .
$$


Let $X_{R} \subset X$ be the quotient of $\Gamma \backslash Y_{R}$. In particular, $X_{1}=X_{\text {cusp. We will write }} T^{1} Y$ for the restriction of the unit tangent bundle to any subset $Y \subset X$. An elementary calculation shows that

$$
\ell\left(T^{1} X_{R}\right)=\left(\frac{1}{R}\right) \frac{\ell\left(T^{1} X_{\text {cusp }}\right)}{\ell\left(T^{1} X\right)}=\frac{C_{X}}{R}
$$

where to simplify notation, henceforth we set $\ell\left(T^{1} X_{\text {cusp }}\right) / \ell\left(T^{1} X\right)$ to be $C_{X}$. Let $\chi_{R}$ be the characteristic function of $T^{1} X_{R}$ and let

Note that

$$
\phi_{R}=\chi_{R / 2}-\chi_{R}
$$

$$
\left\|\phi_{R}\right\|_{L^{1}}=\frac{C_{X}}{R} \text { and }\left\|\phi_{R}\right\|_{L^{2}}=\frac{\sqrt{C_{X}}}{\sqrt{R}}
$$

During an excursion of size at least $R$, a geodesic $\gamma$ must cross $T^{1} X_{R / 2} \backslash T^{1} X_{R}$ twice during a complete excursion and at least once during a partial excursion. By basic hyperbolic geometry, the geodesic spends time greater than $\log 2$ each time it crosses $T^{1} X_{R / 2} \backslash T^{1} X_{R}$.

The next proposition allows us to show that along Leb-almost every geodesic ray, for all times $T$ large enough there is at most a single "large" excursion. The proposition is a continuous time refinement of [9, Lemma 2.1] and the proof uses Lemma 2.11.

Proposition 3.2. For any $c>1 / 2$ and for $\ell$-every $v \in T^{1} X$ there exists $T(v)$ such that for all $T>T(v)$

$$
E\left(\gamma, H_{i}\right) \geqslant T(\log T)^{c}
$$

for at most a single $H_{i} \in H_{\gamma, T}$ and where $\gamma$ is the geodesic ray with $v\left(\gamma_{0}\right)=v$.

For the rest of the discussion, let $T_{n}=2^{n}$. Proposition 3.2 follows from the following proposition.

Proposition 3.3. For any $c>1 / 2$ and for $\ell$-every $v \in T^{1} X$ there exists non-negative integer $n(v)$ such that for all $n>n(v)$

$$
E\left(\gamma, H_{i}\right) \geqslant T_{n-1}\left(\log T_{n-1}\right)^{c}
$$

for at most single $H_{i} \in H_{\gamma, T_{n}}$ and where $\gamma$ is the geodesic ray with $v\left(\gamma_{0}\right)=v$.

Proof. Let $\lambda=\log T_{n-1}+c \log \log T_{n-1}$. By basic hyperbolic geometry, the time a geodesic takes to go from the boundary of a horoball to $X_{R}$ where $R=T_{n-1}\left(\log T_{n-1}\right)^{c}$ is bounded between $\lambda$ and $\log \left(T_{n-1}\left(\log T_{n-1}\right)^{c}+\sqrt{T_{n-1}^{2}\left(\log T_{n-1}\right)^{2 c}-1}\right)<\lambda+\log 2$. Similarly, let $\lambda^{\prime}=\lambda-\log 2$. Then $\lambda^{\prime}$ is a lower bound on the time it takes a geodesic to go from the boundary of a horoball to $X_{R / 2}$ where $R=T_{n-1}\left(\log T_{n-1}\right)^{c}$.

For positive integers $j \leqslant\left\lfloor T_{n} / \lambda\right\rfloor$, let $S_{j}=j \lambda$. Let

$$
V_{n, k}=\left\{v \in T^{1} X \text { such that } \int_{S_{k}}^{S_{k+2}+\lambda^{\prime}} \phi_{R}\left(v\left(\gamma_{s}\right)\right) d s>\log 2 \text { and } \int_{S_{k+2}+\lambda^{\prime}}^{T_{n}} \phi_{R}\left(v\left(\gamma_{t}\right)\right) d t>\log 2\right\} \text {. }
$$

By applying Chebysheff's inequality to the estimate in Lemma 2.11 for the function $\phi_{R}$ we get

$$
\begin{aligned}
\ell\left(V_{n, k}\right) & \leqslant \frac{\left(S_{k+2}+\lambda^{\prime}-S_{K}\right)\left(T_{n}-S_{k+2}-\lambda^{\prime}\right)}{(\log 2)^{2}} \frac{C_{X}^{2}}{R^{2}}+\frac{1}{(\log 2)^{2}}\left(\frac{5 K C_{X}}{\rho R}\left[1-\frac{C_{X}}{R}\right]\right) \\
& <\frac{6 \lambda C_{X}^{2}}{(\log 2)^{2} T_{n-1}\left(\log T_{n-1}\right)^{2 c}}+\frac{5 K C_{X}}{\rho(\log 2)^{2} T_{n-1}\left(\log T_{n-1}\right)^{c}} .
\end{aligned}
$$

In the last inequality we have used $T_{n}-S_{k+2}-\lambda^{\prime}<T_{n}=2 T_{n-1}$.

Let $W$ be the set of $v \in T^{1} X$ such that the corresponding geodesic $\gamma$ has two excursions $E\left(\gamma, H_{i}\right)$ and $E\left(\gamma, H_{j}\right)$ till time $T_{n}$ satisfying $E\left(\gamma, H_{i}\right) \geqslant T_{n-1}\left(\log T_{n-1}\right)^{c}$ and $E\left(\gamma, H_{j}\right) \geqslant T_{n-1}\left(\log T_{n-1}\right)^{c}$. Let $S$ be the time at which the first big excursion $E\left(\gamma, H_{i}\right)$ begins. Let $k$ be such that $S_{k} \leqslant S<S_{k+1}$. 
Because of our choice of $\lambda$ the first excursion $E\left(\gamma, H_{i}\right)$ must end after $S_{k+2}$. Thus, the second big excursion $E\left(\gamma, H_{j}\right)$ also has to begin after $S_{k+2}$. Because of the choice of $\lambda^{\prime}$ the geodesic cannot cross $T^{1} X_{R / 2} \backslash T^{1} X_{R}$ during $E\left(\gamma, H_{j}\right)$ before $S_{k+2}+\lambda^{\prime}$. Thus, $v \in V_{n, k}$. Let

$$
V_{n}=\bigcup_{k=0}^{\left\lfloor T_{n} / \lambda\right\rfloor-2} V_{n, k} .
$$

Using the upper bound on $\ell\left(V_{n, k}\right)$ we get

$$
\begin{aligned}
\ell\left(V_{n}\right) \leqslant \sum_{k=1}^{\left\lfloor T_{n} / \lambda\right\rfloor} \ell\left(V_{n, k}\right) & <\frac{6 T_{n} C_{X}^{2}}{(\log 2)^{2} T_{n-1}\left(\log T_{n-1}\right)^{2 c}}+\frac{5 K T_{n} C_{X}}{\rho(\log 2)^{2} \lambda T_{n-1}\left(\log T_{n-1}\right)^{c}} \\
& <\frac{b_{1} T_{n}}{T_{n-1}\left(\log T_{n-1}\right)^{2 c}}+\frac{b_{2} T_{n}}{T_{n-1}\left(\log T_{n-1}\right)^{1+c}} \\
& \leqslant \frac{2 b_{1}}{\left(\log T_{n-1}\right)^{2 c}}+\frac{2 b_{2}}{\left(\log T_{n-1}\right)^{1+c}}
\end{aligned}
$$

for some constants $b_{1}, b_{2}>0$. Since $c>1 / 2$ it follows that

$$
\sum_{n} \ell\left(V_{n}\right)<\infty
$$

Proposition 3.3 then follows by the Borel-Cantelli lemma.

Proof of Proposition 3.2. Let $n$ be such that $T_{n-1}<T \leqslant T_{n}$. Since $E(\gamma, H) \geqslant T(\log T)^{c}$ implies $E(\gamma, H)>T_{n-1}\left(\log T_{n-1}\right)^{c}$, Proposition 3.2 follows from Proposition 3.3.

Remark 3.4. It is important to observe that Proposition 3.3 holds under the weaker condition that there is a constant $A>1$ such that for $R$ sufficiently large

$$
\frac{1}{A R}<\ell\left(T^{1} X_{R}\right)<\frac{A}{R}
$$

This observation will be of relevance for a similar proposition in the setting of quadratic differentials.

Recall that $x_{0}$ is a base-point and $T_{x_{0}}^{1} X$ can be identified with $S^{1}$.

Corollary 3.5. For any $c>1 / 2$ and Leb-almost every $r \in S^{1}$ there is $T(r)$ such that if $T>T(r)$ then

$$
E\left(\gamma, H_{i}\right) \geqslant T(\log T)^{c}
$$

for at most single $H_{i} \in H_{\gamma, T}$ and where $\gamma$ is the geodesic ray from $x_{0}$ to $r$.

Proof. It follows from Proposition 3.2 that the corollary is true for generic base-points. Suppose $\gamma_{0}$ and $\gamma_{1}$ are geodesic rays from distinct base-points $x_{0}$ and $x_{1}$ converging to the same point $r$ at infinity and let $H$ be a horoball. Let $\pi_{H}$ be the closest point projection to $H$ and let $a=$ $d_{\partial H}\left(\pi_{H} x_{0}, \pi_{H} x_{1}\right)$. Then we have the crude bound

$$
E\left(\gamma_{0}, H\right)-2 a e^{-\tau}-2 \leqslant E\left(\gamma_{1}, H\right) \leqslant E(\gamma, H)+2 a e^{-\tau}+2,
$$

where $\tau$ is the minimum of $d\left(x_{0}, H\right)$ and $d\left(x_{1}, H\right)$. So for $H$ that is far enough the excursions by $\gamma_{0}$ and $\gamma_{1}$ are the same up to a uniform additive constant. This implies the corollary.

Define the function $\psi: X \rightarrow \mathbb{R}$ by

$$
\psi(x)=\left\{\begin{array}{cl}
0 & \text { if } x \in X_{\text {thick }} \\
\left(\frac{2}{\pi}\right) e^{d\left(x, \partial X_{\text {thick }}\right)} & \text { otherwise. }
\end{array}\right.
$$

Let $\Psi: T^{1} X \rightarrow \mathbb{R}$ be the lift of $\psi$ to $T^{1} X$. By definition, the function $\Psi$ is $S O(2, \mathbb{R})$-invariant. 
Suppose a geodesic ray $\gamma$ has a complete excursion in a horoball $H$ entering and exiting $H$ at times $T_{1}$ and $T_{2}$ respectively. We relate the time integral of $\Psi$ between $T_{1}$ and $T_{2}$ to $E(\gamma, H)$.

Conjugating if necessary, we may assume that $H$ is the horoball $\{z: \operatorname{Im}(z) \geqslant 1\}$ in the upper half-space model. The function $\psi$ then takes the form $\psi(z)=(2 / \pi) \operatorname{Im}(z)$ on $H$ and 0 otherwise. Conjugating further by $z \rightarrow z+a$ for some $a \in \mathbb{R}$ we may assume that $\gamma$ is the geodesic with endpoints $\{A,-A\} \in \mathbb{R}$ for some $A \geqslant 1$. We parameterize $\gamma$ with unit speed from $A$ to $-A$. Then, as complex numbers $\gamma\left(T_{1}\right)=\sqrt{A^{2}-1}+i$ and $\gamma\left(T_{2}\right)=-\sqrt{A^{2}-1}+i$. Note that $E(\gamma, H)=$ $2 \sqrt{A^{2}-1}$. Let $\theta_{0}=\operatorname{Arg}\left(\gamma\left(T_{1}\right)\right)$. Then $\operatorname{Arg}\left(\gamma\left(T_{2}\right)\right)=\pi-\theta_{0}$. Using elementary integration, it follows that

$$
\int_{T_{1}}^{T_{2}} \Psi\left(v\left(\gamma_{t}\right)\right) d t=\int_{\theta_{0}}^{\pi-\theta_{0}} \frac{2 A}{\pi} d \theta=\frac{2 A}{\pi}\left(\pi-2 \theta_{0}\right)=2 A-\frac{4 A \theta_{0}}{\pi}
$$

Since $\sin \theta_{0}=1 / A$, it follows that

$$
E(\gamma, H)-2<\int_{T_{1}}^{T_{2}} \Psi\left(v\left(\gamma_{t}\right)\right) d t<E(\gamma, H)+2
$$

i.e., for complete excursions the time integral of $\Psi$ is $E(\gamma, H)$ up to a uniform additive error.

To analyze a partial excursion, we parameterize $\gamma$ by $A e^{i \theta}$ between $\theta_{0}$ and $\pi-\theta_{0}$. Suppose $\theta \in\left(\theta_{0}, \pi-\theta_{0}\right)$. Then the partial excursion till $A e^{i \theta}$ is given by

$$
E(\gamma, H)=A\left(\cos \theta_{0}-\cos \theta\right) .
$$

On the other hand, the time integral is given by

$$
\int \Psi\left(v\left(\gamma_{t}\right)\right) d t=\frac{2 A}{\pi}\left(\theta-\theta_{0}\right),
$$

where $T_{3}<T$ are the times such that $\gamma_{T_{3}}=A e^{i \theta_{0}}$ and $\gamma_{T}=A e^{i \theta}$. The difference of the right hand sides above is symmetric about $\pi / 2$ so we may restrict to $\left(\theta_{0}, \pi / 2\right]$. Then the difference has a crude upper bound given by

$$
A\left(\frac{2}{\pi}\left(\theta-\theta_{0}\right)-\left(\cos \theta_{0}-\cos \theta\right)\right) \leqslant \frac{2 A}{\pi} \theta \leqslant A \sin \theta .
$$

Note that $\Psi\left(v\left(\gamma_{\theta}\right)\right)=(2 / \pi) \operatorname{Im}\left(A e^{i \theta}\right)=(2 / \pi) A \sin \theta$. It follows that for a partial excursion

$$
E(\gamma, H)-\frac{\pi}{2} \Psi\left(v\left(\gamma_{T}\right)\right)<\int_{T_{3}}^{T} \Psi\left(v\left(\gamma_{t}\right)\right) d t<E(\gamma, H)+\frac{\pi}{2} \Psi\left(v\left(\gamma_{T}\right)\right),
$$

where $T_{3}<T$ is the time at which the excursion begins.

Recall that for $\chi_{R}$ is the characteristic function of $T^{1} X_{R}$. We define truncations of $\Psi$ by

$$
\Psi_{R}(v)=\Psi(v)\left(\chi_{1}(v)-\chi_{R}(v)\right),
$$

where $\chi_{1}$ is the characteristic function of $T^{1} X_{1}=T^{1} X_{\text {cusp. }}$. Note that while $\Psi$ is not $L^{1}$ the truncations $\Psi_{R}$ satisfy

$$
\left\|\Psi_{R}\right\|_{L^{1}}=\frac{2 C_{X}}{\pi} \log R \text { and }\left\|\Psi_{R}\right\|_{L^{2}}=\frac{2 \sqrt{C_{X}}}{\pi} \sqrt{R} .
$$

Inequalities 3.6 and 3.7 show that partial sums of excursions (minus the largest excursion) i.e., the numerator in Theorem 1.2 is estimated by the time integral of a suitable truncation of $\Psi$ up to an additive error that is linear in the number $N$ of excursions. The next lemma shows that $N$ grows linearly in $T$.

Lemma 3.8. There is a constant $\eta>0$ such that for Leb-almost every $r \in S^{1}$

$$
\lim _{T \rightarrow \infty} \frac{N}{T}=\eta
$$


Proof of Lemma 3.8. The lemma follows from an approach similar to Schmidt's theorem in the theory of Diophantine approximation [1, Theorem 1.1 with $k=1$ ]. For completeness, we give a weaker but more direct proof below.

For $v \in T^{1}\left(X \backslash X_{\text {thick }}\right)$, let $\gamma$ be the geodesic such that $v\left(\gamma_{0}\right)=v$ i.e., the geodesic whose unit tangent vector at $t=0$ is $v$. For almost every $v$, the geodesic $\gamma$ intersects $\partial X_{\text {thick }}$ both in the forward and backward directions. Let $T_{b}<0$ and $T_{f}>0$ be the first instances of these intersections, i.e. the first instances backward and forward along $\gamma$ when it intersects the boundary of the horoball containing $\pi(v)$. Set

$$
\xi(v)=\frac{1}{T_{f}-T_{b}} .
$$

This defines a non-negative function $\xi: T^{1}\left(X \backslash X_{\text {thick }}\right) \rightarrow \mathbb{R}_{\geqslant 0}$ which we extend by setting it zero outside. It is straightforward to see that for almost every $r \in S^{1}$

$$
\int_{0}^{T} \xi\left(v\left(\gamma_{t}\right)\right) d t=N
$$

We claim that $\xi \in L^{1}\left(T^{1} X\right)$. Let $R_{k}=1+\left(1 / 2^{k}\right)$ and consider $T^{1} X_{R_{k}} \backslash T^{1} X_{R_{k-1}}$. Since $\log (1+$ $\left.\left(1 / 2^{k}\right)\right)=\left(1 / 2^{k}\right)-\left(1 / 2^{2 k+1}\right)+$ higher order terms there exists a constant $b_{3}>0$ such that

$$
\ell\left(T^{1} X_{R_{k-1}} \backslash T^{1} X_{R_{k}}\right)<\frac{b_{3}}{2^{k}}
$$

for all $k$ large enough. By basic hyperbolic geometry, for any $v \in T^{1} X_{R_{k}} \backslash T^{1} X_{R_{k-1}}$

$$
\begin{aligned}
\xi(v) & \leqslant \frac{1}{2 \log \left(1+\left(1 / 2^{k}\right)+\sqrt{\left(1+\left(1 / 2^{k}\right)\right)^{2}-1}\right)} \\
& <\frac{1}{2 \log \left(1+\sqrt{1 / 2^{k}}+\left(1 / 2^{k}\right)\right)} \\
& <b_{4} 2^{k / 2}
\end{aligned}
$$

for some constant $b_{4}>0$ and all $k$ large enough. This gives the bound

$$
\int_{T^{1} X_{R_{k}} \backslash T^{1} X_{R_{k-1}}} \xi d \ell<\frac{b_{3} b_{4}}{2^{k / 2}} .
$$

which proves the claim that $\xi \in L^{1}$. The lemma follows by applying the ergodic theorem to $\xi$.

Proof of Theorem 1.2. While not necessary, for notational simplicity we set the constants $c>1 / 2$ in Proposition 3.2 and in Theorem 2.4 to be equal. Consider the sequence of functions $\Psi_{2^{k}}$. For $T$ such that $2^{k} \leqslant T<2^{k+1}$ we set $n(T)=\left\lfloor k+c \log _{2} k\right\rfloor$ where \lfloor\rfloor is the greatest integer function. By Theorem 2.4 applied to the sequence of functions $\Psi_{2^{n}}$, we have that for any $c>1 / 2$ and $m>1$, $\ell$-almost every $v=v\left(\gamma_{0}\right) \in T^{1} X$ satisfies the lower bound

$$
\frac{1}{m} T\left\|\Psi_{2^{n}}\right\|_{L^{1}}-T^{1 / 2}(\log T)^{c}\left(\left\|\Psi_{2^{n}}\right\|_{L^{2}}^{2}-\left\|\Psi_{2^{n}}\right\|_{L^{1}}^{2}\right)^{1 / 2} \leqslant \int_{0}^{T} \Psi_{2^{n}}\left(v\left(\gamma_{t}\right)\right) d t
$$

and the upper bound

$$
\int_{0}^{T} \Psi_{2^{n}}\left(v\left(\gamma_{t}\right)\right) d t \leqslant m T\left\|\Psi_{2^{n}}\right\|_{L^{1}}+T^{1 / 2}(\log T)^{c}\left(\left\|\Psi_{2^{n}}\right\|_{L^{2}}^{2}-\left\|\Psi_{2^{n}}\right\|_{L^{1}}^{2}\right)^{1 / 2}
$$

for all $T$ large enough depending on $v$ and $m$. Let $r$ be the ratio $T(\log T)^{c} / 2^{n}$. Then $1<r<3$. Substituting the $L^{1}$ and $L^{2}$ norms of $\Psi_{2^{n}}$ in the term $T^{1 / 2}(\log T)^{c}\left(\left\|\Psi_{2^{n}}\right\|_{L^{2}}^{2}-\left\|\Psi_{2^{n}}\right\|_{L^{1}}^{2}\right)^{1 / 2}$, we get 
the upper bound

$$
T^{1 / 2}(\log T)^{c}\left(\frac{4 C_{X}}{r \pi^{2}} T(\log T)^{c}-\frac{4 C_{X}^{2}}{\pi^{2}}(\log T+c \log \log T-\log r)^{2}\right)^{1 / 2} \leqslant \frac{2 \sqrt{C_{X}}}{\pi \sqrt{r}} T(\log T)^{3 c / 2} .
$$

Thus the preceding pair of inequalities become

$$
\begin{aligned}
& \frac{2 C_{X}}{m \pi} T(\log T+c \log \log T-\log r)-\frac{2 \sqrt{C_{X}}}{\pi \sqrt{r}} T(\log T)^{3 c / 2} \leqslant \int_{0}^{T} \Psi_{2^{n}}\left(v\left(\gamma_{t}\right)\right) d t \\
& \int_{0}^{T} \Psi_{2^{n}}\left(v\left(\gamma_{t}\right)\right) d t \leqslant \frac{2 m C_{X}}{\pi} T(\log T+c \log \log T-\log r)+\frac{2 \sqrt{C_{X}}}{\pi \sqrt{r}} T(\log T)^{3 c / 2}
\end{aligned}
$$

We choose $c<2 / 3$. Let $U_{m}$ be the full measure set in $T^{1} X$ satisfying 3.9 and 3.10. Consider the countable intersection

$$
U=\bigcap_{a \in \mathbb{N}} U_{1+1 / a}
$$

Then $U$ has full measure and for $v$ in $U$ the constraint $c<2 / 3$ implies

$$
\lim _{T \rightarrow \infty} \frac{1}{T \log T} \int_{0}^{T} \Psi_{2^{n}}\left(v\left(\gamma_{t}\right)\right) d t=\frac{2 C_{X}}{\pi} .
$$

By the same reasoning as in the proof of Corollary 3.5 the above limit is true for any base-point $x_{0}$ and Leb-almost every $r \in S^{1}$.

It remains to relate the time integral of $\Psi_{2^{n}}$ to partial sum of excursions. Enumerate the horoballs in $\mathcal{H}_{\gamma, T}$ as $H_{1}, \ldots, H_{N}$ in the order of increasing time. For a complete excursion $E\left(\gamma, H_{i}\right)$ satisfying $E\left(\gamma, H_{i}\right) \leqslant T(\log T)^{c}$, the difference between $E\left(\gamma, H_{i}\right)$ and the time integral of $\Psi_{2^{n}}$ between the entry and exit points in $H_{i}$ is at most 2 by 3.6. This implies that the additive error contributed by complete excursions less than the threshold is bounded above by $2 N$.

If $E\left(\gamma, H_{i}\right)>T(\log T)^{c}$ then by Corollary 3.5 it is the unique excursion that exceeds the threshold. Let $T_{1}<T_{2}$ be the entry and exit times in $H_{i}$. In this case, note that

$$
\int_{T_{1}}^{T_{2}} \Psi_{2^{n}}\left(v\left(\gamma_{t}\right)\right) d t<2 T(\log T)^{c} .
$$

If there is a partial excursion then let $T_{3}<T$ be the time at which $\gamma$ enters $H_{N}$. If the partial excursion exceeds the threshold then notice that

$$
\int_{T_{3}}^{T} \Psi_{2^{n}}\left(v\left(\gamma_{t}\right)\right) d t<2 T(\log T)^{c} .
$$

Otherwise, by 3.7 the difference between the time integral and the partial excursion is at most $(\pi / 2) \Psi\left(v\left(\gamma_{T}\right)\right)$ which in turn is bounded above by $T(\log T)^{c}$.

Using the estimates above and also 3.6 and 3.7 we get

$$
\begin{aligned}
\int_{0}^{T} \Psi_{2^{n}}\left(v\left(\gamma_{t}\right)\right) d t-2 N-4 T(\log T)^{c} & <E(\gamma, T)-\max _{1 \leqslant k \leqslant N} E\left(\gamma, H_{k}\right) \\
& <\int_{0}^{T} \Psi_{2^{n}}\left(v\left(\gamma_{t}\right)\right) d t+2 N+4 T(\log T)^{c} .
\end{aligned}
$$

Theorem 1.2 then follows from putting together 3.11, 3.12 and Lemma 3.8.

Proof of Diamond-Vaaler theorem 1.3. For the modular surface $X=S L(2, \mathbb{Z}) \backslash \mathbb{H}^{2}$ the lift to $\mathbb{H}^{2}$ of the largest embedded cusp neighborhood in $X$ is the well-known Ford packing: in the upper half space model, we get horoballs resting at rational points, the Euclidean radius of the horoball with the point at infinity $p / q$ in reduced form being $1 / 2 q^{2}$. 
With the cusp neighborhood fixed as above, Theorem 1.2 for $X$ states that for any base-point and Leb-almost every $r \in S^{1}$

$$
\lim _{T \rightarrow \infty} \frac{E(\gamma, T)-\max _{k \leqslant N} E\left(\gamma, H_{k}\right)}{T \log T}=\frac{6}{\pi^{2}} .
$$

where $\gamma$ is the geodesic ray from some base-point $x_{0}$ to $r$. To derive the Diamond-Vaaler result (Theorem 1.3) from the above limit, we relate excursions to continued fraction coefficients of $r$ and time $T$ along the geodesic to the number $n$ of continued fraction coefficients.

In the upper half space model, for $r \in[0,1]$ irrational, let $\left[a_{1}, \cdots, a_{n}, \cdots\right]$ be the infinite continued fraction expansion of $r$. Let $p_{n} / q_{n}=\left[a_{1}, \ldots, a_{n}\right]$ be the $n$-th convergent of $r$ and let $H_{n}^{\prime}$ be the horoball with $p_{n} / q_{n}$ as the point at infinity. We first consider vertical geodesics: for $r \in[0,1]$ let $\gamma^{\prime}$ be the vertical geodesic ray from $(r, i) \in \mathbb{H}^{2}$ to $(r, 0) \in S^{1}=\mathbb{R} \cup \infty$.

The ray $\gamma^{\prime}$ has excursions in horoballs that are given by rational approximations of $r$ satisfying $|r-p / q| \leqslant 1 / 2 q^{2}$. By a classical theorem for continued fractions, such rationals are a subset of the convergents $p_{n} / q_{n}$. If $a_{n} \geqslant 2$ then $a_{n}-1<E\left(\gamma^{\prime}, H_{n}^{\prime}\right)<a_{n}+1$. However, if $a_{n}=1$ then $\gamma^{\prime}$ may or may not intersect $H_{n}^{\prime}$ and we set $E\left(\gamma^{\prime}, H_{n}^{\prime}\right)=0$ if it does not. In any case, excursions of $\gamma^{\prime}$ are equal to the coefficients up to a uniform additive error and hence we get

$$
\sum_{k=1}^{n} E\left(\gamma^{\prime}, H_{k}^{\prime}\right)-n \leqslant \sum_{k=1}^{n} a_{k} \leqslant \sum_{k=1}^{n} E\left(\gamma^{\prime}, H_{k}^{\prime}\right)+n
$$

By classical theory of continued fractions ([8, Proposition 4.8.2(4)]) for Leb-almost every $r$

$$
\lim _{n \rightarrow \infty} \frac{\log q_{n}}{n}=\frac{\pi^{2}}{12 \log 2}
$$

Since $p_{n} / q_{n} \rightarrow r$, the same limit is true for $\log p_{n} / n$. Up to a transposition of columns, the matrix $Q_{n}$ with columns $\left[p_{n-1}, q_{n-1}\right]^{t}$ and $\left[p_{n}, q_{n}\right]^{t}$ is in $S L(2, \mathbb{Z})$. The hyperbolic translation length of the matrix up to a uniform additive error is $2 \log ($ trace $)$. By the above discussion $\log$ (trace) is $\log q_{n}$ up to a uniform additive error. So let $2 \log q_{n}=T_{n}$. Recall that $Q_{n}$ acts on the upper half plane by Mobius transformations. Geometrically $Q_{n}(r, i)$ is the orbit point closest to $\gamma_{T_{n}}^{\prime}$ with the distance between them bounded above by the diameter of $X_{\text {thick }}$, i.e. uniformly bounded from above. This implies that along the sequence of times $2 \log q_{n}=T_{n}$ the limit $n / T_{n}$ is $6 \log 2 / \pi^{2}$. It should be pointed out that the number $N$ of horoballs that $\gamma^{\prime}$ actually intersects till $T_{n}$ is less than or equal to $n$, and in fact $N / T_{n}$ will have a different limit as $T_{n} \rightarrow \infty$.

The geodesic ray $\gamma$ from $x_{0}$ to $r$ and the vertical ray $\gamma^{\prime}$ are asymptotic. Set

$$
a=\max _{H \in \mathcal{H}} d_{\partial H}\left(\pi_{H} x_{0}, \pi_{H}(r, i)\right)
$$

where $\pi_{H}$ is the closest point projection to $H$. Then we have the crude bound

$$
E(\gamma, H)-2 a e^{-\tau}-2<E\left(\gamma^{\prime}, H\right)<E(\gamma, H)+2 a e^{-\tau}+2
$$

where $\tau$ is minimum of $d\left(x_{0}, H\right)$ and $d((r, i), H)$. Let $d$ be the distance between horocycles with $r$ at infinity that pass through $x_{0}$ and $(r, i)$ respectively. Then depending on the case we get the crude bound

$$
E\left(\gamma, T_{n} \pm d\right)-2(a+1)\left(n+2 \eta\left(T_{n}+d\right)\right) \leqslant \sum_{k \leqslant n} E\left(\gamma^{\prime}, H_{k}^{\prime}\right) \leqslant E\left(\gamma, T_{n} \pm d\right)+2(a+1)\left(n+2 \eta\left(T_{n}+d\right)\right) .
$$

The estimate above implies that

$$
\lim _{n \rightarrow \infty} \frac{\sum_{k \leqslant n} E\left(\gamma^{\prime}, H_{k}^{\prime}\right)-\max _{k \leqslant n} E\left(\gamma^{\prime}, H_{k}^{\prime}\right)}{T \log T}=\lim _{T_{n} \rightarrow \infty} \frac{E\left(\gamma, T_{n} \pm d\right)-\max _{k \leqslant N} E\left(\gamma, H_{k}\right)}{T_{n} \log T_{n}}=\frac{6}{\pi^{2}}
$$


where the second equality follows from the fact that passing to $T_{n}$ instead $T_{n} \pm d$ in the numerator introduces an additive error that is at most $e^{d}$. Finally, note that $a_{k}-1 \leqslant E\left(\gamma^{\prime}, H_{k}^{\prime}\right) \leqslant a_{k}+1$ and so for Leb-almost every $r \in[0,1]$

$$
\begin{aligned}
\lim _{n \rightarrow \infty} \frac{\sum_{k=1}^{n} a_{k}-\max _{k \leqslant n} a_{k}}{n \log n} & =\lim _{n \rightarrow \infty}\left(\frac{\sum_{k \leqslant n} E\left(\gamma^{\prime}, H_{k}^{\prime}\right)-\max _{k \leqslant n} E\left(\gamma^{\prime}, H_{k}^{\prime}\right)}{T_{n} \log T_{n}}\right)\left(\frac{T_{n} \log T_{n}}{n \log n}\right) \\
& =\left(\frac{6}{\pi^{2}}\right)\left(\frac{\pi^{2}}{6 \log 2}\right)=\frac{1}{\log 2}
\end{aligned}
$$

finishing the proof of Theorem 1.3.

\section{PARTial SUms AlONG RANDOM TEICHMÜLler GEODESICS IN A STRATUM OF QUADRATiC DIFFERENTIALS}

4.1. Preliminaries from Teichmüller theory. Let $S$ be a hyperbolic surface of finite type, i.e. a surface of finite area which may have boundary components or punctures. We say such a surface $S$ is sporadic if it is a sphere with at most four punctures or boundary components, or a torus with at most one puncture or boundary component. We shall primarily be interested in non-sporadic surfaces, as in the sporadic cases the Teichmüller spaces are either trivial, or isometric to $\mathbb{H}^{2}$, which reduces us to the case of a non-uniform lattice in $S L(2, \mathbb{R})$.

Let $S$ be a non-sporadic surface which has no boundary components, but may have punctures. The Teichmüller space $\mathcal{T}(S)$ is the space of marked conformal structures on $S$. Alternatively, by uniformization, it is the space of marked hyperbolic metrics on $S$. We shall consider $\mathcal{T}(S)$ together with the Teichmüller metric

$$
d_{\mathcal{T}}(X, Y)=\frac{1}{2} \inf _{f} \log K(f)
$$

where the infimum is taken over all quasiconformal maps $f: X \rightarrow Y$, and $K(f)$ is the quasiconformal constant for the map $f$. The mapping class group $\operatorname{Mod}(S)$ acts by isometries on $\mathcal{T}(S)$. Let $\mathcal{T}(S)_{\epsilon}$ be the thin part of Teichmüller space, i.e. all surfaces which contain a curve of hyperbolic length at most $\epsilon$. Let $\mathcal{M}(S)$ be the moduli space $\operatorname{Mod}(S) \backslash \mathcal{T}(S)$. The thin part $\mathcal{T}(S)$ is $\operatorname{Mod}(S)$ invariant. The thin part $\mathcal{M}(S)_{\epsilon}$ of moduli space is the quotient $\operatorname{Mod}(S) \backslash \mathcal{T}(S)_{\epsilon}$.

Let $\mathcal{Q}(X)$ be the unit area meromorphic quadratic differentials on $X$ with simple poles at all the punctures of $X$. If $\left(k_{1}, k_{2}, \ldots, k_{r}\right)$ are the multiplicities of the zeros of a quadratic differential $q$ then $k_{1}+\cdots+k_{r}=4 g-4+2 m$ where $m$ is the number of punctures of $X$. By contour integration, a quadratic differential $q$ defines a half-translation structure on $S$, i.e. it defines charts from $S$ to $\mathbb{C}$ with transition functions of the form $z \rightarrow \pm z+c$. The resulting flat metric has a cone singularity with cone angle $(k+2) \pi$ at a zero of $q$ order $k$ (or with $k=-1$ at a simple pole). A quadratic differential is unit area if the corresponding flat metric has unit area. The space $\mathcal{Q}$ of unit area quadratic differentials can be identified with the unit cotangent bundle to $\mathcal{T}(S)$ [16]. We let $\pi: \mathcal{Q} \rightarrow \mathcal{T}(S)$ be the projection which sends a quadratic differential to its underlying Riemann surface. The space $\mathcal{Q}$ is stratified by the multiplicities of the zeros: we denote the strata with multiplicities $\alpha=\left(k_{1}, \ldots, k_{r}\right)$ by $\mathcal{Q}(\alpha)$. For each stratum, the number of connected components is bounded. See [18], [7]. To simplify notation, we continue to denote $\mathcal{Q}(\alpha)$ when we mean a connected component of $\mathcal{Q}(\alpha)$.

For any $q \in \mathcal{Q}(\alpha)$ there is a canonical ramified double cover such that the lift of $q$ is square of a holomorphic 1-form $\omega$ and $(X, q)$ is a quotient of the double cover with respect to hyper-elliptic involution. Fixing a basis for the anti-invariant (with respect to hyper-elliptic involution) part of the homology of the double cover relative to the singularities, the holonomies (periods) given by 
integrating $\omega$ over the basis defines local co-ordinates on $\mathcal{Q}(\alpha)$. The natural volume form in these co-ordinates defines the Masur-Veech measure. Alternatively, it is known as the holonomy measure. We shall denote it by $\mu_{\text {hol }}$. The measure $\mu_{\text {hol }}$ is $\operatorname{Mod}(S)$-invariant. So it descends to a measure on $\operatorname{Mod}(S) \backslash \mathcal{Q}(\alpha)$, the corresponding stratum of the moduli space of quadratic differentials. We

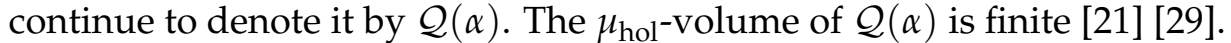

The affine action of $S L(2, \mathbb{R})$ on the charts to $\mathbb{C}=\mathbb{R}^{2}$ preserves the glueing by half-translations. This defines an action of $S L(2, \mathbb{R})$ on $\mathcal{Q}(\alpha)$. The orbits $S L(2, \mathbb{R})(q)$ foliate $\mathcal{Q}(\alpha)$. The compact part $S O(2, \mathbb{R})$ acts by rotations of $\mathbb{R}^{2}$. Hence, it preserves the conformal structure. The action of the diagonal subgroup defines the Teichmüller geodesic flow. It shrinks the leaves of the vertical foliation for $q$ and stretches the leaves of the horizontal foliation for $q$ by the same factor. It follows from the definition that $\mu_{\text {hol }}$ is $S L(2, \mathbb{R})$-invariant.

Since $S O(2, \mathbb{R})$ preserves the conformal structure we get an isometrically embedded $\mathbb{H}^{2}=$ $S L(2, \mathbb{R}) / S O(2, R)$ in $\mathcal{T}(S)$. This is called a Teichmüller disc and we will denote the Teichmüller disc determined by $q$ as $\mathbb{D}(q)$. The Teichmüller metric restricted to $\mathbb{D}(q)$ is isometric to the hyperbolic plane of constant curvature -4 .

In the flat metric defined by a quadratic differential $q$, a saddle connection is a geodesic segment that connects a pair of (same or distinct) singularities of $q$. The $\epsilon$-thin part, $\mathcal{Q}(\alpha)_{\epsilon}$ of $\mathcal{Q}(\alpha)$ is the subset of $q$ such that $\ell_{q}^{2}(\beta) \leqslant \epsilon$ for some saddle connection $\beta$.

The points $q^{\prime}$ in $S L(2, \mathbb{R})(q)$ where $\ell_{q^{\prime}}^{2}(\beta) \leqslant \epsilon$ projects to a horoball in $\mathbb{D}(q)$. The point at infinity of the horoball is given by the direction in which $\beta$ is vertical. When two saddle connections $\beta_{1}$ and $\beta_{2}$ are parallel the proportion $\left[\ell_{q^{\prime}}\left(\beta_{1}\right): \ell_{q^{\prime}}\left(\beta_{2}\right)\right]$ as a function of $q^{\prime}$ is constant. Hence, the horoball is determined by the saddle connection with the shortest holonomy in a collection of parallel saddle connections. Typically, the intersection $\mathcal{Q}(\alpha)_{\epsilon} \cap \mathbb{D}(q)$ is a complicated collection of horoballs in $\mathbb{D}(q)$ with their interiors having intersections. Every point in $\mathbb{D}(q)$ is contained in at most finitely many horoballs. But there need not be a uniform upper bound over $\mathbb{D}(q)$ for this number. This makes it hard to get traction on the $d_{\text {thick }}$-distance between the entry and exit points of a geodesic in $\mathcal{Q}(\alpha)_{\epsilon} \cap \mathbb{D}(q)$, which would have been a natural analog of an excursion in this context. Instead, we consider excursions in individual horoballs.

The excursions in horoballs in $\mathbb{D}(q)$ of a geodesic $\gamma$ till time $T$ are defined as follows. For a horoball $H$ that $\gamma$ enters and exits, the complete excursion $E(\gamma, H)$ is defined as the distance along $\partial H$ between the entry and exit points. If $\gamma_{T} \in H$ then the partial excursion $E(\gamma, H)$ is defined as the distance along $\partial H$ between the entry point of $\gamma$ and $\pi_{H}\left(\gamma_{T}\right)$ where $\pi_{H}: H \rightarrow \partial H$ is the closest point projection. Let $H_{1}, \ldots, H_{N(T)}$ denote all the horoballs that $\gamma$ intersects till time $T$. The total excursion $E(\gamma, T)$ is defined as

$$
E(\gamma, T)=\sum_{k \leqslant N(T)} E\left(\gamma, H_{k}\right)
$$

When horoballs have disjoint interiors these definitions coincide with the definitions in Section 1.1. With intersections, the excursions $E\left(\gamma, H_{k}\right)$ can be simultaneous and there can be several partial excursions when $\gamma_{T}$ is in the thin part.

4.2. $S L(2, \mathbb{R})$ orbit closures and invariant measures. Recently, Eskin and Mirzakhani [12, Theorem 1.4] showed that ergodic $S L(2, \mathbb{R})$-invariant probability measures are of Lebesgue class and are supported on invariant complex submanifolds in $\mathcal{Q}(\alpha)$. These manifolds are affine in the sense that in the holonomy co-ordinates on $\mathcal{Q}(\alpha)$ they are cut out by linear equations. Going further, Eskin, Mirzakhani and Mohammadi in [13, Theorem 2.1] show that all $S L(2, \mathbb{R})$ orbit closures are affine invariant submanifolds. See [12, Section 1] for more details. More recently, Filip [14] shows that these submanifolds are in fact algebraic subvarieties. 
4.3. Thin parts and regularity for invariant measures. Let $\mu$ be an ergodic $S L(2, \mathbb{R})$-invariant probability supported on an affine invariant submanifold $\mathcal{N} \subset \mathcal{Q}(\alpha)$. For $\epsilon>0$, we define the $\epsilon$ thin part of $\mathcal{N}$ as follows:

$$
\mathcal{N}_{\epsilon}=\left\{q \in \mathcal{N} \text { such that } \ell_{q}^{2}(\beta) \leqslant \epsilon \text { for some saddle connection } \beta\right\} .
$$

Saddle connections $\beta_{1}, \beta_{2}$ are $\mathcal{N}$-parallel if they are parallel for an open subset of quadratic differentials in $\mathcal{N}$. See Definition 4.6 in [31].

Regularity: For $\epsilon>0, \kappa>0$ small enough, let $\mathcal{N}_{\epsilon, \kappa}$ be the subset of $q \in \mathcal{N}$ such that there is a pair of saddle connections $\beta_{1}, \beta_{2}$ not $\mathcal{N}$-parallel such that $\ell_{q}^{2}\left(\beta_{1}\right) \leqslant \epsilon$ and $\ell_{q}^{2}\left(\beta_{2}\right) \leqslant \kappa$. The measure $\mu$ is said to be regular if there exists a constant $m_{1}>0$ such that

$$
\mu\left(\mathcal{N}_{\epsilon, \kappa}\right) \leqslant m_{1} \epsilon \kappa
$$

Masur and Smillie [23, Section 10, Claim (7)] show that the holonomy measure $\mu_{\text {hol }}$ is regular. Avila, Matheus and Yoccoz [4, Theorem 1.2] prove a weaker version of regularity for any $S L(2, \mathbb{R})$ invariant measure.

4.5. $S L(2, \mathbb{R})$-invariant loci, Siegel-Veech transform and volume asymptotic. For $q \in \mathcal{N}$, let $V(q) \subset \mathbb{R}^{2} \backslash\{(0,0)\}$ be an assignment of a non-empty subset of holonomies of saddle connections on $q$. We require that the assignment varies linearly under $S L(2, \mathbb{R})$ action, i.e. $V(g q)=g V(q)$ for all $g \in S L(2, \mathbb{R})$. As observed in [10], such an assignment satisfies conditions $(B)$ and $C_{\mu}$ mentioned in their paper for any $S L(2, \mathbb{R})$-invariant measure $\mu$. Such an assignment $V$ will be called a $S L(2, \mathbb{R})$-invariant locus.

Let $f$ be a smooth function on $\mathbb{R}^{2}$ with compact support. The Siegel-Veech transform associated to a $S L(2, \mathbb{R})$-invariant locus $V$ is defined as

$$
\widehat{f}(q)=\sum_{v \in V(q)} f(v)
$$

Veech showed that $f \in L^{1}(\mathcal{N}, \mu)$ and proved the Siegel-Veech formula

$$
\int_{\mathcal{N}} \widehat{f} d \mu=c(V, \mu) \int_{\mathbb{R}^{2}} f d x d y
$$

where the constant $c(V, \mu)$ does not depend on $f$. The constant $c(V, \mu)$ is called the Siegel-Veech constant associated to $V$ and $\mu$. We assume that the assignment $V$ is such that $c(V, \mu)>0$.

For $R \geqslant 1$, the $\epsilon / R$-thin part of $\mathcal{N}$ corresponding to $V$ is the set of $q$ with some saddle connection with holonomy in $V$ has $q$-length squared less than $\epsilon / R$. We denote the set by $\mathcal{N}(V)_{\epsilon / R}$.

Let $f_{\epsilon / R}$ be the characteristic function of the ball $B((0,0), \sqrt{\epsilon / R})$ centered at the origin and radius $\sqrt{\epsilon / R}$. While $f_{\epsilon / R}$ is not smooth the Siegel-Veech formula extends to such characteristic functions. The regularity condition 4.4 and the Siegel-Veech formula 4.6 applied to $f_{\epsilon / R}$ can be used to prove the volume asymptotic

$$
\lim _{R \rightarrow \infty} \frac{\mu\left(\mathcal{N}(V)_{\epsilon / R}\right)}{\pi \epsilon / R}=c(V, \mu) .
$$

See [11, Section 7] for the main ideas.

4.8. Exponential mixing of Teichmüller flow: It is known that the Teichmüller flow is exponentially mixing. For the Masur-Veech measure, the decay of correlations 3.1 for $S O(2, \mathbb{R})$-invariant $L^{2}$-functions is due to Avila-Gouëzel-Yoccoz [3] for holomorphic 1-forms and due to Avila-Resende [5] for quadratic differentials. For general $S L(2, \mathbb{R})$-invariant measures this is due to Avila-Gouëzel [2]. Since the functions we consider are pullbacks from Teichmüller discs they are $S O(2, \mathbb{R})$ invariant. Hence, the decay of correlations applies to them. 


\section{Proofs of Theorem 1.9}

The simplest case: We first prove Theorem 1.9 in the case when $V(q)$ is the set of holonomies of all saddle connections on $q$. This allows us to convey the key ideas while getting into fewer subtleties. We denote the corresponding Siegel-Veech constant simply as $c(\mu)$.

Let $q \in \mathcal{N}_{\epsilon}$. Consider short saddle connections in $q$. If some saddle connections are parallel we choose the one with the smallest holonomy among them. Suppose that lengths of these short saddle connections are given by $\ell_{q}^{2}\left(\beta_{1}\right)=\epsilon / R_{1}, \ell_{q}^{2}\left(\beta_{2}\right)=\epsilon / R_{2}, \ldots, \ell_{q}^{2}\left(\beta_{k}\right)=\epsilon / R_{k}$ with $R_{1} \geqslant$ $R_{2} \geqslant \cdots \geqslant R_{k} \geqslant 1$. We define

$$
\Psi(q)=\frac{2}{\pi} R_{1}
$$

Next we define

$$
\bar{\Psi}(q)=\frac{2}{\pi}\left(R_{1}+R_{2}+\cdots+R_{k}\right) .
$$

Obviously $\bar{\Psi}(q) \geqslant \Psi(q)$ for all $q$. At first glance, the function $\Psi$ above is similar to the function $\Psi$ defined in the context of non-uniform lattices. However, here there can be simultaneous excursions. Hence, the sum over all excursions between successive entry and exit times $T_{1}<T_{2}$ in $\mathcal{N}_{\epsilon}$ can satisfy

$$
\sum_{H: \gamma\left[T_{1}, T_{2}\right] \cap H \neq \varnothing} E(\gamma, H) \gg \int_{T^{1}}^{T_{2}} \Psi\left(v\left(\gamma_{t}\right)\right) d t .
$$

This discrepancy is rectified by using the larger function $\bar{\Psi}$. The key point is to estimate the difference in the $L^{1}$ and $L^{2}$ norms of the truncations of $\Psi$ and $\Psi$ in terms of the depth in $\mathcal{N}_{\epsilon}$ of the truncations. This will enable us to show that the above discrepancy does not happen too often.

For times $T$ when $\gamma_{T}$ is in the thick part, it would be interesting to relate the integral over time of $\bar{\Psi}$ to $d_{\text {thick }}\left(\gamma_{0}, \gamma_{T}\right)$. In analogy with the observations preceding Theorem 1.5, we expect their ratio to be asymptotic to a constant $M>0$ that depends only on $\mathcal{N}$.

Let $\chi_{R}$ denote the characteristic function of $\mathcal{N}_{\epsilon / R}$ and define the truncation

$$
\Psi_{R}=\left(\chi_{1}-\chi_{R}\right) \Psi
$$

\section{Lemma 5.1.}

$$
\lim _{R \rightarrow \infty} \frac{\left\|\Psi_{R}\right\|_{L^{1}}}{\log R}=2 \epsilon c(\mu), \lim _{R \rightarrow \infty} \frac{\left\|\Psi_{R}\right\|_{L^{2}}}{\sqrt{R}}=2 \frac{\sqrt{\epsilon c(\mu)}}{\sqrt{\pi}}
$$

Proof. It follows from 4.7 that for any $A>1$ there is $R_{0}$ such that for all $R>R_{0}$

$$
\frac{1}{A} \frac{\pi \epsilon c(\mu)}{R}<\mu\left(\mathcal{N}_{\epsilon / R}\right)<A \frac{\pi \epsilon c(\mu)}{R} .
$$

Fix $r>0$ and for any positive integer $k$ consider $\mathcal{N}_{\epsilon / 2^{(k-1) r}} \backslash \mathcal{N}_{\epsilon / 2^{k r}}$. If $k$ is large enough so that $2^{(k-1) r}>R_{0}$ then the measure of the above set satisfies

$$
\frac{\pi \epsilon c(\mu)}{2^{k r}}\left(\frac{2^{r}-A^{2}}{A}\right)<\mu\left(\mathcal{N}_{\epsilon / 2^{(k-1) r}} \backslash \mathcal{N}_{\epsilon / 2^{k r}}\right)<\frac{\pi \epsilon c(\mu)}{2^{k r}}\left(\frac{2^{r} A^{2}-1}{A}\right) .
$$

Given $r$, we choose $A$ close to 1 such that

$$
\frac{2^{r}-1}{2^{r}}<\frac{2^{r}-A^{2}}{A}<\frac{2^{r} A^{2}-1}{A}<2^{r}\left(2^{r}-1\right)
$$


Let $n$ be the largest integer such that $2^{n r} \leqslant R$. The $L^{1}$-norm of $\Psi_{R}$ can be estimated by

$$
\begin{aligned}
\frac{2}{\pi} \sum_{k=1}^{n} 2^{(k-1) r} \mu\left(\mathcal{N}_{\epsilon / 2^{(k-1) r}} \backslash \mathcal{N}_{\epsilon / 2^{k r}}\right) & <\left\|\Psi_{R}\right\|_{L^{1}} \\
& <\frac{2}{\pi} \sum_{k=1}^{n+1} 2^{k r} \mu\left(\mathcal{N}_{\epsilon / 2^{(k-1) r}} \backslash \mathcal{N}_{\epsilon / 2^{k r}}\right) .
\end{aligned}
$$

Let $n_{0}$ be the smallest integer such that $2^{n_{0} r} \geqslant R_{0}$. We assume that $R \gg R_{0}$. The summation in the lower bound on the left satisfies

$$
\begin{aligned}
& \sum_{k=0}^{n} 2^{(k-1) r} \mu\left(\mathcal{N}_{\left.\epsilon / 2^{(k-1) r} \backslash \mathcal{N}_{\epsilon / 2^{k r}}\right)>} \sum_{k=1}^{n_{0}-1} 2^{(k-1) r} \mu\left(\mathcal{N}_{\epsilon / 2^{(k-1) r}} \backslash \mathcal{N}_{\epsilon / 2^{k r}}\right)+\right. \\
& \sum_{k=n_{0}}^{n} 2^{(k-1) r} \frac{\pi \epsilon c(\mu)}{2^{k r}}\left(\frac{2^{r}-1}{2^{r}}\right) .
\end{aligned}
$$

The right hand side of the above inequality simplifies to

$$
\sum_{k=1}^{n_{0}-1} 2^{(k-1) r} \mu\left(\mathcal{N}_{\epsilon / 2^{(k-1) r}} \backslash \mathcal{N}_{\epsilon / 2^{k r}}\right)+\frac{\left(n-n_{0}\right) \pi \epsilon c(\mu)}{2^{r}}\left(\frac{2^{r}-1}{2^{r}}\right) .
$$

As $R$ becomes large, the second term dominates and since $\left(n-n_{0}\right) / \log R \rightarrow 1 / r \log 2$ the above expression simplifies to

which as $r \rightarrow 0$ implies

$$
\frac{2}{2^{2 r} \log 2}\left(\frac{2^{r}-1}{r}\right) \epsilon c(\mu)<\lim _{R \rightarrow \infty} \frac{\left\|\Psi_{R}\right\|_{L^{1}}}{\log R}
$$

$$
2 \epsilon c(\mu) \leqslant \lim _{R \rightarrow \infty} \frac{\left\|\Psi_{R}\right\|_{L^{1}}}{\log R} .
$$

Similarly the summation for the upper bound on $\left\|\Psi_{R}\right\|_{L^{1}}$ gives

$$
\frac{2}{2^{r} \log 2}\left(\frac{2^{r}-1}{r}\right) \epsilon c(\mu)>\lim _{R \rightarrow \infty} \frac{\left\|\Psi_{R}\right\|_{L^{1}}}{\log R}
$$

which as $r \rightarrow 0$ implies

$$
2 \epsilon c(\mu) \geqslant \lim _{R \rightarrow \infty} \frac{\left\|\Psi_{R}\right\|_{L^{1}}}{\log R}
$$

proving the lemma for the $L^{1}$-norm.

In a similar way, the square of the $L^{2}$-norm of $\Psi_{R}$ can be estimated by

$$
\begin{aligned}
\frac{4}{\pi^{2}} \sum_{k=1}^{n} 2^{2(k-1) r} \mu\left(\mathcal{N}_{\epsilon / 2^{(k-1) r}} \backslash \mathcal{N}_{\epsilon / 2^{k r}}\right) & <\left\|\Psi_{R}\right\|_{L^{2}}^{2} \\
& <\frac{4}{\pi^{2}} \sum_{k=1}^{n+1} 2^{2 k r} \mu\left(\mathcal{N}_{\epsilon / 2^{(k-1) r}} \backslash \mathcal{N}_{\epsilon / 2^{k r}}\right) .
\end{aligned}
$$

The summation in the lower bound on the left satisfies

$$
\begin{aligned}
\sum_{k=1}^{n} 2^{2(k-1) r} \mu\left(\mathcal{N}_{\epsilon / 2^{(k-1) r}} \backslash \mathcal{N}_{\epsilon / 2^{k r}}\right)> & \sum_{k=1}^{n_{0}-1} 2^{2(k-1) r} \mu\left(\mathcal{N}_{\epsilon / 2^{(k-1) r}} \backslash \mathcal{N}_{\epsilon / 2^{k r}}\right)+ \\
& \sum_{k=n_{0}}^{n} 2^{2(k-1) r} \frac{\pi \epsilon c(\mu)}{2^{k r}}\left(\frac{2^{r}-1}{2^{r}}\right) .
\end{aligned}
$$


The right hand side of the inequality above is equal to

$$
\sum_{k=1}^{n_{0}-1} 2^{2(k-1) r} \mu\left(\mathcal{N}_{\epsilon / 2^{(k-1) r}} \backslash \mathcal{N}_{\epsilon / 2^{k r}}\right)+\left(\frac{2^{(n+1) r}-2^{n_{0} r}}{2^{r}-1}\right) \frac{\pi \epsilon c(\mu)}{2^{2 r}}\left(\frac{2^{r}-1}{2^{r}}\right)
$$

which is greater than

$$
\sum_{k=1}^{n_{0}-1} 2^{2(k-1) r} \mu\left(\mathcal{N}_{\epsilon / 2^{(k-1) r}} \backslash \mathcal{N}_{\epsilon / 2^{k r}}\right)+\frac{\pi \epsilon c(\mu) R}{2^{3 r}}-\frac{2^{n_{0} r} \pi \epsilon c(\mu)}{2^{3 r}} .
$$

As $R$ becomes large the term containing $R$ dominates and letting $r \rightarrow 0$ we get the lower bound

$$
\frac{2 \sqrt{\epsilon c(\mu)}}{\sqrt{\pi}} \leqslant \lim _{R \rightarrow \infty} \frac{\left\|\Psi_{R}\right\|_{L^{2}}}{\sqrt{R}} .
$$

Similarly the summation in the upper bound satisfies

$$
\begin{aligned}
\sum_{k=1}^{n+1} 2^{k r} \mu\left(\mathcal{N}_{\epsilon / 2^{(k-1) r}} \backslash \mathcal{N}_{\epsilon / 2^{k r}}\right)< & \sum_{k=1}^{n_{0}-1} 2^{2 k r} \mu\left(\mathcal{N}_{\epsilon / 2^{(k-1) r}} \backslash \mathcal{N}_{\epsilon / 2^{k r}}\right)+ \\
& \sum_{k=n_{0}}^{n+1} 2^{2 k r} \frac{\pi \epsilon c(\mu)}{2^{(k-1) r}}\left(2^{r}\left(2^{r}-1\right)\right) \\
& <\sum_{k=1}^{n_{0}-1} 2^{2 k r} \mu\left(\mathcal{N}_{\epsilon / 2^{(k-1) r}} \backslash \mathcal{N}_{\epsilon / 2^{k r}}\right)+2^{4 r} R \pi \epsilon c(\mu) .
\end{aligned}
$$

As $R$ becomes large the term containing $R$ dominates and letting $r \rightarrow 0$ we get the upper bound

$$
\frac{2 \sqrt{\epsilon c(\mu)}}{\sqrt{\pi}} \geqslant \lim _{R \rightarrow \infty} \frac{\left\|\Psi_{R}\right\|_{L^{2}}}{\sqrt{R}} .
$$

finishing the proof for the $L^{2}$-norm.

The next lemma will need the regularity in 4.4 of $\mu$ and the following theorem of Eskin and Masur: For any stratum $\mathcal{Q}(\alpha)$ and any $0<\delta<1$ there exists constants $m_{2}(\alpha, \delta)>0$ such that the number of saddle connections shorter than $\kappa<1$ is bounded above by

$$
s(q) \leqslant m_{2}\left(\frac{\kappa}{\ell_{q}(\beta)}\right)^{1+\delta}
$$

where $\beta$ is the shortest saddle connection for $q$. It should be noted that while Eskin and Masur state the theorem for strata of holomorphic 1-forms it is also true for strata of quadratic differentials by passing to the canonical double cover.

Fix the constant $\delta$ in the Eskin-Masur theorem and choose $a>1$ such that $a<2 /(1+\delta)$. Let $\mathcal{N}^{\prime} \subset \mathcal{N}_{\epsilon / R} \backslash \mathcal{N}_{\epsilon / R^{a}}$ be the subset of quadratic differentials such that apart from the shortest saddle connection, all other short saddle connections satisfy $\ell_{q}^{2}\left(\beta_{i}\right) \geqslant \epsilon / R$. We define the function $\Psi^{\prime}: \mathcal{N}^{\prime} \rightarrow \mathbb{R}_{\geqslant 0}$ by

$$
\Psi^{\prime}(q)=\frac{2}{\pi}\left(R_{2}+R_{3}+\cdots+R_{j}\right) .
$$

We define a slightly more complicated truncation

$$
\bar{\Psi}_{R}=\bar{\Psi}\left(\chi_{1}-\chi_{R}\right)+\Psi^{\prime} .
$$

In this particular case of Theorem 1.9, the extra term $\Psi^{\prime}$ allows us to keep track of excursions that are concurrent with the largest excursion if it exceeds $T(\log T)^{c}$. 
Lemma 5.4. There exists a constant $B>0$ such that for $R$ large enough

$$
\left\|\bar{\Psi}_{R}\right\|_{L^{1}} \leqslant\left\|\Psi_{R}\right\|_{L^{1}}+B \text {. }
$$

The $L^{2}$-norms satisfy

$$
\left\|\bar{\Psi}_{R}\right\|_{L^{2}} \leqslant\left\|\Psi_{R}\right\|_{L^{2}}+o(\sqrt{R}) .
$$

Proof. Consider the subset $\mathcal{N}_{k, j}$ of $\mathcal{N}_{\epsilon / 2^{k-1}} \backslash \mathcal{N}_{\epsilon / 2^{k}}$ of those $q$ such that the length of the second shortest saddle connection $\beta_{2}$ (not $\mathcal{N}$-parallel to $\beta_{1}$ ) satisfies $\epsilon / 2^{j-1} \geqslant \ell_{q}^{2}\left(\beta_{2}\right)>\epsilon / 2^{j}$ where $j \leqslant k$. By regularity 4.4,

$$
\mu\left(\mathcal{N}_{k, j}\right) \leqslant \frac{m_{1} \epsilon^{2}}{2^{k-1} 2^{j-1}} .
$$

For a quadratic differential $q$ in $\mathcal{N}_{k, j}$, using the bound 5.3 on the number of short saddle connections we get the pointwise bound

$$
\bar{\Psi}_{R}(q)-\Psi_{R}(q)<\sum_{i=1}^{j} 2^{i} m_{2}\left(\frac{2^{k}}{2^{i-1}}\right)^{(1+\delta) / 2}<2 m_{2} 2^{k(1+\delta) / 2} \sum_{i=1}^{j} 2^{(i-1)(1-\delta) / 2}<m_{3} 2^{k(1+\delta) / 2} 2^{j(1-\delta) / 2}
$$

for some constant $m_{3}>0$. Thus

$$
\int_{\mathcal{N}_{k, j}}\left(\bar{\Psi}_{R}-\Psi_{R}\right) d \mu<\left(m_{3} 2^{k(1+\delta) / 2} 2^{j(1-\delta) / 2}\right)\left(\frac{m_{1} \epsilon^{2}}{2^{k-1} 2^{j-1}}\right)=\frac{4 m_{1} m_{3} \epsilon^{2}}{2^{k(1-\delta) / 2} 2^{j(1+\delta) / 2}} .
$$

Summing over $j=1$ to $k$ (when $2^{k-1}>R$ it suffices to sum till the smallest number $k^{\prime}$ such that $2^{k^{\prime}}>R$ in which case the sum would be even smaller) we get

$$
\int_{\mathcal{N}_{\epsilon / 2^{k-1}} \backslash \mathcal{N}_{\epsilon / 2^{k}}}\left(\bar{\Psi}_{R}-\Psi_{R}\right) d \mu<\frac{m_{4}}{2^{k(1-\delta) / 2}}
$$

for some constant $m_{4}>0$. Let $n_{a}$ be the smallest integer such that $2^{n_{a}} \geqslant R^{a}$. The bound for the integral established above implies that

$$
\left\|\bar{\Psi}_{R}\right\|_{L^{1}}-\left\|\Psi_{R}\right\|_{L^{1}}<\sum_{k=1}^{n_{a}} \frac{m_{4}}{2^{k(1-\delta) / 2}} .
$$

The sum on the right hand side is bounded from above independent of $n_{a}$ which proves the lemma for $L^{1}$ norms.

The same pointwise bound above implies

$$
\begin{aligned}
\int_{\mathcal{N}_{k, j}} \bar{\Psi}_{R}^{2} d \mu & <\int_{\mathcal{N}_{k, j}} \Psi_{R}^{2} d \mu+2 m_{3} 2^{k(1+\delta) / 2} 2^{j(1-\delta) / 2} \int_{\mathcal{N}_{k, j}} \Psi_{R} d \mu+m_{3}^{2} 2^{k(1+\delta)} 2^{j(1-\delta)} \mu\left(\mathcal{N}_{k, j}\right) \\
& <\int_{\mathcal{N}_{k, j}} \Psi_{R}^{2} d \mu+2 m_{3} 2^{k(1+\delta) / 2} 2^{j(1-\delta) / 2}\left(\frac{2^{k} m_{1} \epsilon^{2}}{2^{k-1} 2^{j-1}}\right)+m_{3}^{2} 2^{k(1+\delta)} 2^{j(1-\delta)} \frac{m_{1} \epsilon^{2}}{2^{k-1} 2^{j-1}} \\
& =\int_{\mathcal{N}_{k, j}} \Psi_{R}^{2} d \mu+\frac{1}{2^{j(1+\delta) / 2}} 8 m_{1} m_{3} \epsilon^{2} 2^{k(1+\delta) / 2}+\frac{1}{2^{j \delta}} 4 m_{1} m_{3}^{2} \epsilon^{2} 2^{k \delta}
\end{aligned}
$$

Summing over $j=1$ to $k$ we get

$$
\int_{\mathcal{N}_{\epsilon / 2^{k-1}} \backslash \mathcal{N}_{\epsilon / 2^{k}}} \Psi_{R}^{2} d \mu<\int_{\mathcal{N}_{\epsilon / 2^{k-1}} \backslash \mathcal{N}_{\epsilon / 2^{k}}} \Psi_{R}^{2} d \mu+m_{5} 2^{k(1+\delta) / 2}+m_{6} 2^{k \delta}
$$

for some constants $m_{5}, m_{6}>0$. Summing over $k=1$ to $n_{a}$ we get

$$
\left\|\bar{\Psi}_{R}\right\|_{L^{2}}^{2}<\left\|\Psi_{R}\right\|_{L^{2}}^{2}+\frac{m_{7}}{2} 2^{n_{a}(1+\delta) / 2}+\frac{m_{8}}{2} 2^{n_{a} \delta}<\left\|\Psi_{R}\right\|_{L^{2}}^{2}+m_{7} R^{a(1+\delta) / 2}+m_{8} R^{a \delta}
$$


for some constants $m_{7}, m_{8}>0$. Recall that we had chosen $a>1$ to satisfy $a(1+\delta)<2$ which implies $a \delta<a(1+\delta) / 2<1$. Thus, the corresponding terms on the right hand side are $o(R)$ from which the lemma follows for $L^{2}$-norms.

We will justify the choice of the cutoff $R^{a}$ used for truncation by the following sharper lemma. The lemma is a special case of a continuous time version of the analog of Borel-Bernstein theorem [17, Theorem 30], [1].

Lemma 5.5. For any $1 / 2<c<1$ and $\mu$-almost every $q \in \mathcal{N}$ there is $T_{0}$ depending on $q$ such that for all $T>T_{0}$, all excursions $E(\gamma, H)$ till time $T$ satisfy

$$
E(\gamma, H)<T(\log T)^{2 c}
$$

Proof. We recall some notation. For $R \geqslant 1$, we denote by $\phi_{R}$ the characteristic function of $\mathcal{N}_{2 \epsilon / R} \backslash$ $\mathcal{N}_{\epsilon / R}$. Choose $A$ close to 1 so that estimate 5.2 is satisfied with $r=1$ i.e., $A>1$ is chosen close enough to 1 such that

$$
\frac{1}{2}<\frac{2-A^{2}}{A}<\frac{2 A^{2}-1}{A}<2
$$

The given choice of $A$ implies that for all $R$ large enough

$$
\left\|\phi_{R}\right\|_{L^{1}}<\frac{2 \pi \epsilon c(\mu)}{R} \text { and hence }\left\|\phi_{R}\right\|_{L^{2}}^{2}<\frac{2 \pi \epsilon c(\mu)}{R} \text {. }
$$

Define the function $n: \mathbb{R} \rightarrow \mathbb{N}$ such that for $T$ satisfying $2^{k} \leqslant T<2^{k+1}$ the positive integers $n(T)$ is the largest integer such that $2^{n} \leqslant k^{2 c} 2^{k}$ i.e., $n=\lfloor k+2 c \log k\rfloor$. Choose a constant $c_{1}>1 / 2$ for Theorem 2.4 to satisfy $c_{1}<c$. Fixing $m>1$, the upper bound in Theorem 2.4 implies that for $\mu$-almost every $q$

$$
\int_{0}^{T} \phi_{2^{n}}\left(v\left(\gamma_{t}\right)\right) d t \leqslant m T\left\|\phi_{2^{n}}\right\|_{L^{1}}+T^{1 / 2}(\log T)^{c_{1}}\left(\left\|\phi_{2^{n}}\right\|_{L^{2}}^{2}-\left\|\phi_{2^{n}}\right\|_{L^{1}}^{2}\right)^{1 / 2}
$$

for all $T$ large enough depending on $q$ and $m$ and with $n(T)$ defined as above. By passing to a larger $T$ if necessary, we can ensure that the upper bounds above are also satisfied by $\left\|\phi_{2^{n}}\right\|_{L^{1}}$ and $\left\|\phi_{2^{n}}\right\|_{L^{2}}^{2}$. This gives the upper bound

$$
\begin{aligned}
\int_{0}^{T} \phi_{2^{n}}\left(v\left(\gamma_{t}\right)\right) d t & \leqslant m T \frac{2 \pi \epsilon c(\mu)}{2^{n}}+T^{1 / 2}(\log T)^{c_{1}}\left(\frac{2 \pi \epsilon c(\mu)}{2^{n}}\right)^{1 / 2} \\
& <\frac{B_{1}}{(\log T)^{2 c}}+\frac{B_{2}}{(\log T)^{c-c_{1}}}
\end{aligned}
$$

for some constants $B_{1}, B_{2}>0$. Notice that if $T$ is large enough the right hand side is less than $\log 2$. But if an excursion till time satisfies $E(\gamma, H) \geqslant T(\log T)^{2 c}$ then $\gamma$ must spend time at least $\log 2$ in $\mathcal{N}_{\epsilon / 2^{n-1}} \backslash \mathcal{N}_{\epsilon / 2^{n}}$, which proves the lemma.

Proof of Theorem 1.9 when $V$ is all saddle connection holonomies. Fix $c$ satisfying $1 / 2<c<1$. Observe that Proposition 3.2 holds in this setting. That is, along $\mu$-typical geodesics there is at most a single excursion larger than $T(\log T)^{c}$ till time $T$ for $T$ large enough. In fact, as noted in Remark 3.4, the proof of Proposition 3.3 does not need the precise asymptotic for $\mu\left(\mathcal{N}_{\epsilon / R}\right)$ as $R \rightarrow \infty$, just that $\mu\left(\mathcal{N}_{\epsilon / R}\right)$ decays as $1 / R$ up to some multiplicative constant.

If $2^{k} \leqslant T<2^{k+1}$, then let $n=n(T)=\left\lfloor k+c \log _{2} k\right\rfloor$. Replicating the exact argument in the proof of Theorem 1.2, we use Lemma 5.1 to conclude that for $\mu$-almost every $q \in \mathcal{N}$

$$
\lim _{T \rightarrow \infty} \frac{1}{T \log T} \int_{0}^{T} \Psi_{2^{n}}\left(v\left(\gamma_{t}\right)\right) d t=2 \epsilon c(\mu)
$$


where $\gamma$ is the Teichmüller geodesic ray with $v\left(\gamma_{0}\right)=q$. Lemma 5.4 implies that the above limit holds when $\Psi_{2^{n}}$ is replaced by $\bar{\Psi}_{2^{n}}$. By Lemma 5.5, for $T$ large enough the largest excursion till time $T$ is smaller than $T(\log T)^{2 c}<T^{a}$. Hence, up to an additive error whose dependence on $T$ will be described below

$$
E(\gamma, T)-\max _{k \leqslant N} E\left(\gamma, H_{k}\right) \asymp \int_{0}^{T} \bar{\Psi}_{2^{n}}\left(v\left(\gamma_{t}\right)\right) d t .
$$

The additive error is bounded above by the sum of the additive errors arising from individual excursions. First, we analyze the partial excursions (if they exists).

The partial excursions correspond to configurations of saddle connections that are short in $q_{T}=$ $v\left(\gamma_{T}\right)$. If the $q_{T}$-length squared of the small saddle connection in such a configuration is $\epsilon / R$ then by 3.7, the additive error for the associated partial excursion is at most $R$. Let $\beta_{1}$ be the shortest saddle connection in $q_{T}$. Let $\beta_{2}$ be the second shortest non-parallel saddle connection. Lemma 5.5 implies that if $T$ is large enough then $\ell_{q_{T}}^{2}\left(\beta_{1}\right) \geqslant \epsilon / T(\log T)^{2 c}$. Proposition 3.2 implies that $\ell_{q_{T}}^{2}\left(\beta_{2}\right) \geqslant \epsilon / T(\log T)^{c}$. Let $n$ be the smallest positive integer such that $2^{n} \geqslant T(\log T)^{2 c}$. Let $m$ be the smallest positive integer such that $2^{m} \geqslant T(\log T)^{c}$. The Eskin-Masur bound 5.3 implies that the total additive error is bounded above by

$$
\begin{aligned}
\sum_{k=0}^{m-1} 2^{k+1} m_{2}\left(\frac{2^{n / 2}}{2^{k / 2}}\right)^{1+\delta} & =\sum_{k=0}^{m-1} 2 m_{2} 2^{k(1-\delta) / 2} 2^{n(1+\delta) / 2} \\
& \leqslant B_{3} 2^{m(1-\delta) / 2} 2^{n(1+\delta) / 2} \\
& \leqslant 4 B_{3} T^{(1-\delta) / 2}(\log T)^{c(1-\delta) / 2} T^{(1+\delta) / 2}(\log T)^{2 c(1+\delta) / 2} \\
& =4 B_{3} T(\log T)^{c(3+\delta) / 2}
\end{aligned}
$$

for some constant $B_{3}>0$. For this bound to be of a lower order, $c$ must satisfy $c<2 /(3+\delta)$. Note that $2 /(3+\delta)>1 / 2$. So the condition can be satisfied.

By 3.6, the additive error from an individual complete excursion is uniform. This means that the additive error from all complete excursions is at most linear in the number $N$ of horoballs that $\gamma$ intersects till time $T$. We claim that $N$ grows linearly in $T$. Let $\mathcal{S}_{T}$ be the saddle connections for $q$ whose length squared gets shorter than $\epsilon$ in time less than $T$ along the Teichmüller geodesic ray determined by $q$. Then the necessary conditions on the $(x, y)$-coordinates of the holonomy vectors of the saddle connections in $\mathcal{S}_{T}$ are $|x y| \leqslant \epsilon^{2} / 2, y \leqslant e^{T}$ and $x<\epsilon$. By [1, Theorem 1.6] the number of such vectors for a $\mu$-almost every $q$ is linear in $T$ thus proving the claim.

5.6. The general case. Let $V$ be a $S L(2, \mathbb{R})$-invariant locus. Suppose that for $q$ there are $j$ short saddle connections no two of which are $\mathcal{N}$-parallel with holonomy in $V(q)$. Index the saddle connections $\beta_{1}, \beta_{2}, \ldots, \beta_{j}$ in the order of increasing $q$-lengths and let $\ell_{q}^{2}\left(\beta_{1}\right)=\epsilon / R_{1}, \ell_{q}^{2}\left(\beta_{2}\right)=$ $\epsilon / R_{2}, \ldots, \ell_{q}^{2}\left(\beta_{j}\right)=\epsilon / R_{j}$. We define the functions

$$
\Psi^{V}(q)=\frac{2}{\pi} R_{1}
$$

and

$$
\bar{\Psi}^{V}(q)=\frac{2}{\pi}\left(R_{1}+R_{2}+\cdots+R_{j}\right) .
$$

We can define truncations of these functions in an analogous way using the characteristic function of $\mathcal{N}(V)_{\epsilon / R}$. However, the shortest saddle connection $\beta$ for $q$ may not have holonomy in $V(q)$ and be shorter than $\beta_{1}$ as above. Hence, some care is required in defining the truncations. The crucial point is that in light of Lemma 5.5, we can impose a lower bound on the length of $\beta$ in defining 
the truncations. As in the previous case, we use a sloppier lower bound than what Lemma 5.5 implies. This simplifies the expressions in the estimates.

Let $a>1$ be such that $a<2 /(1+\delta)$. In particular, $a \delta<1$. Let $\mathcal{N}(R, a) \subset \mathcal{N}(V)_{\epsilon} \backslash \mathcal{N}(V)_{\epsilon / R}$ be the subset of those $q$ such that the shortest saddle connection $\beta$ satisfies $\ell_{q}^{2}(\beta) \geqslant \epsilon / R^{a}$. Let $\chi_{R, a}$ denote its characteristic function of $\mathcal{N}(R, a)$.

Let $\mathcal{N}^{\prime}(V) \subset \mathcal{N}(V)_{\epsilon / R} \backslash \mathcal{N}(V)_{\epsilon / R^{a}}$ be the subset of quadratic differentials such that $\ell_{q}^{2}(\beta) \geqslant$ $\epsilon / R^{a}$ and apart from $\beta_{1}$ all other short saddle connections with holonomy in $V(q)$ satisfy $\ell_{q}^{2}\left(\beta_{i}\right) \geqslant$ $\epsilon / R$. Let $\left(\Psi^{V}\right)^{\prime}: \mathcal{N}^{\prime}(V) \rightarrow \mathbb{R}$ be defined as

$$
\left(\Psi^{V}\right)^{\prime}(q)=\frac{2}{\pi}\left(R_{2}+\cdots+R_{j}\right)
$$

We define $\Psi_{R}^{V}=\Psi^{V} \chi_{R, a}$ and $\Psi_{R}^{V}=\bar{\Psi}^{V} \chi_{R, a}+\left(\Psi^{V}\right)^{\prime}$. Again, the extra term $\left(\Psi^{V}\right)^{\prime}$ analogous to $\Psi^{\prime}$ earlier, allows us to track excursions for saddle connections in $V$ that happen during the largest excursion with holonomy in $V$ should it exceed $T(\log T)^{c}$.

For $2^{k} \leqslant R$ we have the estimate

$$
\mu\left(\mathcal{N}(V)_{\epsilon / 2^{k-1}} \backslash \mathcal{N}(V)_{\epsilon / 2^{k}}\right)-\mu\left(\mathcal{N}(R, a) \cap \mathcal{N}(V)_{\epsilon / 2^{k-1}} \backslash \mathcal{N}(V)_{\epsilon / 2^{k}}\right) \leqslant \frac{m_{1} \epsilon^{2}}{2^{k-1} R^{a}} .
$$

This means here each term in the summations for lower and upper bound for $L^{1}$-norm in Lemma 5.1 changes by at most $2^{k} m_{1} \epsilon^{2} / 2^{k-1} R^{a}=2 m_{1} \epsilon^{2} / R^{a}$. Hence the summations change by at most $2 n m_{1} \epsilon^{2} / R^{a}<m_{6} \log R / R$ for some constant $m_{6}>0$. This implies

$$
\lim _{R \rightarrow \infty} \frac{\left\|\Psi_{R}^{V}\right\|_{L^{1}}}{\log R}=2 \epsilon c(V, \mu)
$$

Similarly each term in the summations for lower and upper bound for $L^{2}$ norms changes by at most $2^{2 k} m_{1} \epsilon^{2} / 2^{k-1} R^{a}=m_{1} \epsilon^{2} 2^{k+1} / R^{a}$ and hence the summations change by at most $4 m_{1} \epsilon^{2} 2^{n} / R^{a}<$ $m_{7} / R^{a-1}$ for some constant $m_{7}>0$. This implies

$$
\lim _{R \rightarrow \infty} \frac{\left\|\Psi_{R}^{V}\right\|_{L^{2}}}{\sqrt{R}}=2 \frac{\sqrt{\epsilon c(V, \mu)}}{\sqrt{\pi}} .
$$

Lemma 5.7. There exists a constant $B_{V}$ such that for $R$ large enough

$$
\left\|\bar{\Psi}_{R}^{V}\right\|_{L^{1}} \leqslant\left\|\Psi_{R}^{V}\right\|_{L^{1}}+B_{V} .
$$

The $L^{2}$-norms satisfy

$$
\left\|\bar{\Psi}_{R}^{V}\right\|_{L^{2}} \leqslant\left\|\Psi_{R}^{V}\right\|_{L^{2}}+o(\sqrt{R}) .
$$

Proof. Consider $\mathcal{N}(R, a) \cap \mathcal{N}(V)_{\epsilon / 2^{k-1}} \backslash \mathcal{N}(V)_{\epsilon / 2^{k}}$ and let $\mathcal{N}_{k, j}^{V}$ be its subset consisting of those $q$ for which (among the collection of non-parallel saddle connections with holonomies in $V(q)$ ) the second shortest saddle connection $\beta_{2}$ satisfies $\epsilon / 2^{j-1} \geqslant \ell_{q}^{2}\left(\beta_{2}\right)>\epsilon / 2^{j}$ where $j \leqslant k$.

We further partition $\mathcal{N}_{k, j}^{V}$ into two sets $\mathcal{N}_{k, j}^{V}(1) \cup \mathcal{N}_{k, j}^{V}(2)$ depending on whether the shortest saddle connection for $q$ has holonomy in $V(q)$ or not, i.e. $\mathcal{N}_{k, j}^{V}(1)$ is the subset of $q$ for which $\beta_{1}$ is the shortest saddle connection and $\mathcal{N}_{k, j}^{V}(2)$ is when its not. On $\mathcal{N}_{k, j}^{V}(1)$ the integral

$$
\int_{\mathcal{N}_{k, j}^{V}(1)}\left(\bar{\Psi}_{R}^{V}-\Psi_{R}^{V}\right) d \mu
$$

is bounded from above identical to Lemma 5.5. 
Let $n_{a}$ be the smallest integer such that $2^{n_{a}} \geqslant R^{a}$. For $q \in \mathcal{N}_{k, j}^{V}(2)$ suppose that the shortest saddle connection $\beta$ satisfies $\epsilon / 2^{p-1}>\ell_{q}^{2}(\beta) \geqslant \epsilon / 2^{p}$ where $2^{k} \leqslant 2^{p} \leqslant 2^{n_{a}}$. The measure of the subset of such $q$ is bounded above by

$$
\frac{m_{1} \epsilon^{2}}{2^{p-1} 2^{k-1}} .
$$

The number of short saddle connections whose $q$-length squared is at least $\epsilon / 2^{i-1}$ is bounded above by

$$
m_{2}\left(\frac{2^{p}}{2^{i-1}}\right)^{(1+\delta) / 2}
$$

This gives the pointwise bound

$$
\Psi_{R}^{V}(q)-\Psi_{R}^{V}(q)<\sum_{i=1}^{j} 2^{i} m_{2}\left(\frac{2^{p}}{2^{i-1}}\right)^{(1+\delta) / 2}=2 m_{2} 2^{p(1+\delta) / 2} \sum_{i=1}^{j} 2^{(i-1)(1-\delta) / 2}<m_{9} 2^{p(1+\delta) / 2} 2^{j(1-\delta) / 2}
$$

for some constant $m_{9}>0$. This gives the bound

$$
\int_{\mathcal{N}_{k, j}^{V}(2)}\left(\bar{\Psi}_{R}^{V}-\Psi_{R}^{V}\right) d \mu<\sum_{p=k}^{n_{a}} m_{9} 2^{p(1+\delta) / 2} 2^{j(1-\delta) / 2}\left(\frac{m_{1} \epsilon^{2}}{2^{p-1} 2^{k-1}}\right)<\frac{m_{10}}{2^{k}}
$$

for some constant $m_{10}>0$. Thus adding up the upper bounds for the integrals on $\mathcal{N}_{k, j}^{V}(1)$ and $\mathcal{N}_{k, j}^{V}(2)$ we get

$$
\int_{\mathcal{N}_{k, j}^{V}}\left(\bar{\Psi}_{R}^{V}-\Psi_{R}^{V}\right) d \mu<\frac{4 m_{1} m_{3} \epsilon^{2}}{2^{k(1-\delta) / 2} 2^{j(1+\delta) / 2}}+\frac{m_{10}}{2^{k}}
$$

Summing over $j=1$ to $k$ we get

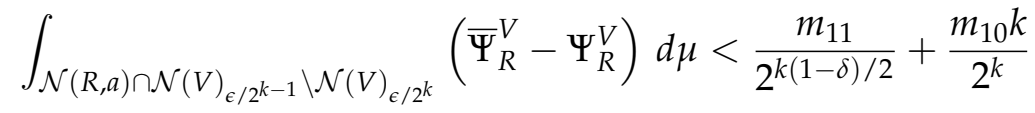

for some constant $m_{11}>0$. Summing over $k=1$ to $n_{a}$ observe that the sum of the right hand side is bounded independent of $n$ which proves the lemma for $L^{1}$-norms.

The pointwise bound also implies

$$
\begin{aligned}
\int_{\mathcal{N}_{k, j}^{V}(2)}\left(\bar{\Psi}_{R}^{V}\right)^{2}-\left(\Psi_{R}^{V}\right)^{2} d \mu< & \sum_{p=k}^{n_{a}} 2 m_{9} 2^{p(1+\delta) / 2} 2^{j(1-\delta) / 2}\left(\frac{2^{k} m_{1} \epsilon^{2}}{2^{p-1} 2^{k-1}}\right) \\
& +\sum_{p=k}^{n_{a}} m_{9}^{2} 2^{p(1+\delta)} 2^{j(1-\delta)}\left(\frac{m_{1} \epsilon^{2}}{2^{p-1} 2^{k-1}}\right) \\
< & \frac{m_{12}}{2^{k}}+\frac{m_{13} 2^{n_{a} \delta}}{2^{k \delta}}
\end{aligned}
$$

for some constants $m_{12}, m_{13}>0$. The corresponding upper bound for $\mathcal{N}_{k, j}^{V}(1)$ is identical to Lemma 5.4 and is of the form

$$
\int_{\mathcal{N}_{k, j}^{V}(1)}\left(\Psi_{R}^{V}\right)^{2}-\left(\Psi_{R}^{V}\right)^{2} d \mu<\frac{m_{14} 2^{k(1+\delta) / 2}}{2^{j(1+\delta) / 2}}+\frac{m_{15} 2^{k \delta}}{2^{j \delta}}
$$

for some constants $m_{14}, m_{15}>0$. Adding up the bounds for $\mathcal{N}_{k, j}^{V}(1)$ and $\mathcal{N}_{k, j}^{V}(2)$ and summing over $j=1$ to $k$ we get

$$
\int_{\mathcal{N}(R, a) \cap \mathcal{N}(V)_{\epsilon / 2^{k-1}} \backslash \mathcal{N}(V)_{\epsilon / 2^{k}}}\left(\bar{\Psi}_{R}^{V}\right)^{2}-\left(\Psi_{R}^{V}\right)^{2} d \mu<\frac{m_{12} k}{2^{k}}+\frac{m_{13} k 2^{n_{a} \delta}}{2^{k \delta}}+m_{14} 2^{k(1+\delta) / 2}+m_{15} 2^{k \delta} .
$$


and when $2^{k-1}>R$,

$$
\int_{\mathcal{N}^{\prime}(V) \cap \mathcal{N}(V)_{\epsilon / 2^{k-1}} \backslash \mathcal{N}(V)_{\epsilon / 2^{k}}}\left(\bar{\Psi}_{R}^{V}\right)^{2}-\left(\Psi_{R}^{V}\right)^{2} d \mu<\frac{m_{12} k}{2^{k}}+\frac{m_{13} k 2^{n_{a} \delta}}{2^{k \delta}}+m_{14^{2}} 2^{k(1+\delta) / 2}+m_{15} 2^{k \delta}
$$

Summing over $k=1$ to $n_{a}$ we get that

$$
\left\|\Psi_{R}^{V}\right\|_{L^{2}}^{2}-\left\|\Psi_{R}^{V}\right\|_{L^{2}}^{2}<\frac{m_{16}}{2} 2^{n_{a} \delta}+\frac{m_{17}}{2} 2^{n_{a}(1+\delta) / 2}+m_{18}<m_{16} R^{a \delta}+m_{17} R^{a(1+\delta) / 2}+m_{18}
$$

for some constants $m_{15}, m_{16}, m_{17}, m_{18}>0$. The condition on $a$ implies that the right hand side is $o(R)$. Thus the lemma follows for $L^{2}$-norms.

Proof of Theorem 1.9. Fix $c$ satisfying $1 / 2<c<1$. As in the earlier case when $V$ is all saddle holonmies, Proposition 3.2 holds for $\mathcal{N}(V)$ for the same reason. It asserts that for $\mu$-almost every $q$, the Teichmüller geodesic ray corresponding to $q$ has at most a single excursion (in horoballs given by saddle connections with holonomies in $V$ ) till $T$ that is larger than $T(\log T)^{c}$ for all $T$ large enough depending on $q$. Moreover, by Lemma 5.5 the largest excursion till time $T$ cannot exceed $T(\log T)^{2 c}$.

The later fact implies that up to additive error our truncation $\bar{\Psi}_{2^{n}}^{V}$ satisfies

$$
\int_{0}^{T} \bar{\Psi}_{2^{n}}^{V}\left(v\left(\gamma_{t}\right)\right) d t \asymp E(\gamma, T)-\max _{k \leqslant N_{V}} E\left(\gamma, H_{k}\right)
$$

where the additive error is of order lower than $T \log T$ for the same reason as earlier. Theorem 2.4 and Lemma 5.7 conclude the proof of Theorem 1.9 in the general case, the precise argument a replica of earlier proofs.

\section{REFERENCES}

[1] Athreya, J., Parrish, A. and Tseng, J. Ergodic theory and diophantine approximation for linear forms and translation surfaces. preprint, (2014), http:/ / arxiv.org/abs/1401.4148.

[2] Avila, A. and Gouëzel, S. Small eigenvalues of the Laplacian for algebraic measures in moduli space, and mixing properties of the Teichmüller flow. Ann. of Math. (2) 178 (2013), no. 2, 385-442.

[3] Avila, A., Gouëzel, S. and Yoccoz, J-C. Exponential mixing for the Teichmüller flow. Publ . Math. Inst. Hautes Etudes Sci. no. 104 (2006), 143-211.

[4] Avila, A., Matheus, C. and Yoccoz, J-P. SL(2, R)-invariant probability measures on the moduli spaces of translation surfaces are regular. Geom. Funct. Anal. 23, (2013), no. 6, 1705-1729.

[5] Avila, A. and Resende, M-J. Exponential mixing for the Teichmüller flow in the space of quadratic differentials. Comment. Math. Helv. 87 (2012), no. 3, 589-638.

[6] Avila, A. and Viana, M. Simplicity of Lyapunov spectra: proof of the Zorich-Kontsevich conjecture. Acta Math. 198 (2007), no. 1, 1-56.

[7] Boissy, C. and Lanneau, E. Dynamics and geometry of Rauzy-Veech induction for quadratic differentials. Ergodic Theory and Dynam. Systems, 29 (2009). no. 3, 767-816.

[8] Brin, M. and Stuck, G. Introduction to Dynamical Systems. Cambridge University Press (2002), xii+240pp. ISBN: 0-521-80841-3.

[9] Diamond, H and Vaaler, J. Estimates for partial sums of continued fraction partial quotients. Pacific. J. Math. 122 (1986), no. 1, 73-82.

[10] Eskin, A. and Masur, H. Asymptotic formulas on flat surfaces. Ergodic Theory Dynam. Systems 21 (2001), no. 2, 443-478.

[11] Eskin, A., Masur, H. and Zorich, A. Moduli spaces of Abelian differentials: The principle boundary, counting problems and the Siegel-Veech constants. Publ. Math. Inst. Hautes. Études. Sci. No 97, (2003), 61-179.

[12] Eskin, A. and Mirzakhani, M. Invariant and stationary measures for the $S L(2, \mathbb{R})$ action on moduli space. preprint.

[13] Eskin, A, Mirzakhani, M. and Mohammadi, A. Isolation, equidistribution and orbit closures for the $S L(2, \mathbb{R})$ action on moduli space. preprint.

[14] Filip, S. Splitting mixed Hodge structures over affine invariant manifolds. preprint. 
[15] Gadre, V., Maher, J. and Tiozzo, G. Word length statistics for Teichmüller geodesics and singularity of harmonic measure. preprint, (2013), http:/ / arxiv.org/abs/1212.1481.

[16] Hubbard, J. and Masur, H. Quadratic differentials and foliations. Acta Math. 142 (1979), no. 3-4, 221-274.

[17] Khintchine, A. Continued fractions. U. Chicago Press, (1964), Chicago IL.

[18] Kontsevich, M. and Zorich, A. Connected components of the moduli space of abelian differentials with prescribed singularities. Invent. Math. 153 (2003), no. 3, 631-678.

[19] Lannaeu, E. Connected components of the strata of the moduli spaces of quadratic differentials. Ann. Sci. Éc. Norm. Supér. (4) 41, (2008), no. 1, 1-56.

[20] Masur, H. Uniquely ergodic quadratic differentials. Comment. Math. Helv. 55 (1980), no. 2, 255-266.

[21] Masur, H. Interval exchange transformations and measured foliations. Ann. of Math. (2) 115 (1982), no. 1, $169-200$.

[22] Masur, H. Logarithm law for geodesics in moduli space. (Göttingen, 1991/ Seattle, WA, 1991) Contemp. Math. vol. 150, Amer. Math. Soc., Providence, RI (1993), 229-245.

[23] Masur, H. and Smillie, J. Hausdorff dimension of sets of nonergodic foliations. Ann. Math. 134, (1991), $455-543$.

[24] Masur, H. and Zorich, A. Multiple saddle connections on flat surfaces and the principle boundary of the moduli space of quadratic differentials. Geom. Funct. Anal. 18, (2008), no. 3, 919-987.

[25] Matheus, C. Some quantitative versions of Ratner's mixing theorems. Bull. Braz. Math. Soc. (N.S) 44 (2013), no. 3, 469488.

[26] Moore, C. Exponential decay of correlation coefficients for geodesic flows. Group Representations, ergodic theory, operator algebras and mathematical physics (Berkeley, Calif., 1984), 163-181, Math. Sci. Res. Inst. Publ., no. 6, Springer, New York, (1987).

[27] Ratner, M. The rate of mixing for geodesic and horocycle flows. Ergodic Theory Dynam. Systems 7, 267-288, (1987).

[28] Sullivan, D. Disjoint spheres, approximation by imaginary quadratic numbers and the logarithm law for geodesics. Acta Math. 3-4 (1982), 215-237.

[29] Veech, W. The Teichmüller geodesic flow. Ann. of Math. (2) 124 (1986), no. 3, 441-530.

[30] Vorobets, Y. Periodic geodesics on generic translation surfaces. Algebraic and topological dynamics, 205-258, Contemp. Math., 385 (2005), Amer. Math. Soc., Providence, RI.

[31] Wright, A. Cylinder deformations in orbit closures of translation surfaces. to appear in Geom. Topol.

MATHEMATICs Institute, ZEemAN BUILDING, UNIVERSITY OF WARWICK, COVENTRY, CV4 7AL.

E-mail address: gadre.vaibhav@gmail.com 